\title{
Transport and Use of a Centaur Second Stage in Space
}

\author{
James M. Strong* \\ NASA Ames Research Center, Moffett Field, CA 94035 \\ Bernard Morgowicz ${ }^{\dagger}$, Eric Drucker ${ }^{\ddagger}$ \\ Northrop Grumman Aerospace Systems, Redondo Beach, CA 90278 \\ Paul D. Tompkins ${ }^{\S}$ \\ Stinger Ghaffarian Technologies, Moffett Field, CA 94035 \\ Brian Kennedy" \\ NASA Jet Propulsion Laboratory, Pasadena, CA 91109-8099 \\ Robert Barber ${ }^{\#}$, Louie T. Luzod ${ }^{* *}$ \\ NASA Ames Research Center, Moffett Field, CA 94035
}

\begin{abstract}
As nations continue to explore space, the desire to reduce costs will continue to grow. As a method of cost reduction, transporting and/or use of launch system components as integral components of missions may become more commonplace in the future. There have been numerous scenarios written for using launch vehicle components (primarily space shuttle used external tanks) as part of flight missions or future habitats. Future studies for possible uses of launch vehicle upper stages might include asteroid diverter using gravity orbital perturbation, orbiting station component, raw material at an outpost, and kinetic impactor. The LCROSS (Lunar CRater Observation and Sensing Satellite) mission was conceived as a low-cost means of determining whether water exists at the polar regions of the moon. Manifested as a secondary payload with the LRO (Lunar Reconnaissance Orbiter) spacecraft aboard an Atlas V launch vehicle, LCROSS guided its spent Centaur Earth Departure Upper Stage (EDUS) into the lunar crater Cabeus, as a kinetic impactor. This paper describes some of the challenges that the LCROSS project encountered in planning, designing, launching with and carrying the Centaur upper stage to the moon.
\end{abstract}

\section{Introduction}

THE primary objective of the Lunar Crater Observation and Sensing Satellite (LCROSS) mission was to advance the Vision for Space Exploration by determining the possible existence of water ice at the lunar poles. Locating sources of water has numerous implications for future missions to the moon. The LCROSS concept originated when the Lunar Reconnaissance Orbiter (LRO) switched to a larger launch vehicle having launch capacity sufficient for a secondary payload. LCROSS was selected from a number of proposals to occupy this extra capacity in part due to innovative use of the Centaur upper stage as a primary impactor, and the judicious use of the Evolved Expendable Launch Vehicle (EELV) Secondary Payload Adaptor (ESPA) ring. The ESPA was originally designed to carry six separate small satellites between the Atlas Centaur upper stage and the primary mission payload (e.g. LRO) ${ }^{1,2}$. For the LCROSS mission, the ESPA ring was used for the shepherding spacecraft's primary

\footnotetext{
${ }^{*}$ Flight Controller, LCROSS, Project Engineering Division, M/S 240A-3, AIAA Senior Member

${ }^{\dagger}$ Lead Attitude Control Engineer, LCROSS, Control Systems Department, M/S R4/1212

${ }^{\ddagger}$ Lead A\&FM Systems Engineer, LCROSS, Space Vehicle Systems Engineering, M/S: M2/2300

§ Lead Flight Director, LCROSS, Intelligent Systems Division (Code TI), M/S 269-3

" Lead Navigation Engineer, LCROSS, Inner Planetary Navigation Group, MS 264-820

\# Project Systems Engineer, LCROSS, Engineering Systems Division, M/S 240-2

** Launch Systems Manager, LCROSS, Flight System Implementation, M/S 240A-3
}

1

American Institute of Aeronautics and Astronautics 
structure with the science payload and spacecraft subsystem panels attached externally to it, while retaining its LRO launch adaptor and load bearing function ${ }^{3}$. The project was managed, partially developed and operated from NASA Ames Research Center (ARC), and the LCROSS spacecraft was designed, built and tested at Northrop Grumman. The Centaur upper stage, developed by United Launch Alliance (ULA), is designed for payload orbital insertions and not for extended operations in space.

LCROSS launched with LRO on June 18, 2009, and on October 9, 2009, after 112 days in flight, both the Centaur upper stage and LCROSS shepherding spacecraft (S-S/C) completed the mission by impacting the moon on target in the crater Cabeus. The mission demonstrated a unique capability by transporting and successfully guiding the Centaur to impact a permanently shadowed crater at the lunar south pole. After the Centaur impact, the S-S/C followed, collecting imagery and spectra of the Centaur impact debris plume for evidence of ice and water vapor, and impacting itself, four minutes later. After one month of post-impact analysis, the LCROSS science team announced a positive identification of water on the floor of the Cabeus crater ${ }^{4}$. LCROSS's use of the $2300 \mathrm{~kg}$ spent upper stage Centaur as a primary kinetic impactor, rather than the more typical use of the spacecraft itself for this purpose, and carrying it for the duration of the mission presented new and unique challenges for the project. The success of LCROSS demonstrated the capability to effectively use a launch system component as an integral part of a short, science mission. The following sections describe the mission briefly and detail some of the unique features this mission was required to accommodate while flying with the Centaur upper stage.

\section{Launch, Mission and Requirements}

LCROSS and LRO launched aboard an Atlas V launch vehicle in the 401configuration (4-meter fairing, no solid rocket boosters, and a hydrogen/oxygen burning single-engine, Centaur upper stage). After the Atlas/Centaur separation (launch plus 4 minutes, 8 seconds), the Centaur performed a 9 minute, 40 second main engine burn achieving low-Earth orbit, followed by a 23 minute coast. The Centaur then performed a 4 minute, 55 second trans-lunar injection (TLI) burn for both LCROSS and LRO, completing 42 minutes after launch. Three minutes after burn completion, LRO separated and the Centaur performed a collision and contamination avoidance maneuver. Having delivered LRO, the Centaur performed an additional $31 / 2$ minute impulsive maneuver and blowdown using its main engine, to deliver LCROSS to its own delivery orbit. Following LRO separation and during Centaur propellant depletion procedures, the Centaur initiated LCROSS activation via a series of six discrete commands, issued over 14 seconds, to trigger primary and redundant stages of the LCROSS power-up sequence (see section III). Importantly, these first six discrete

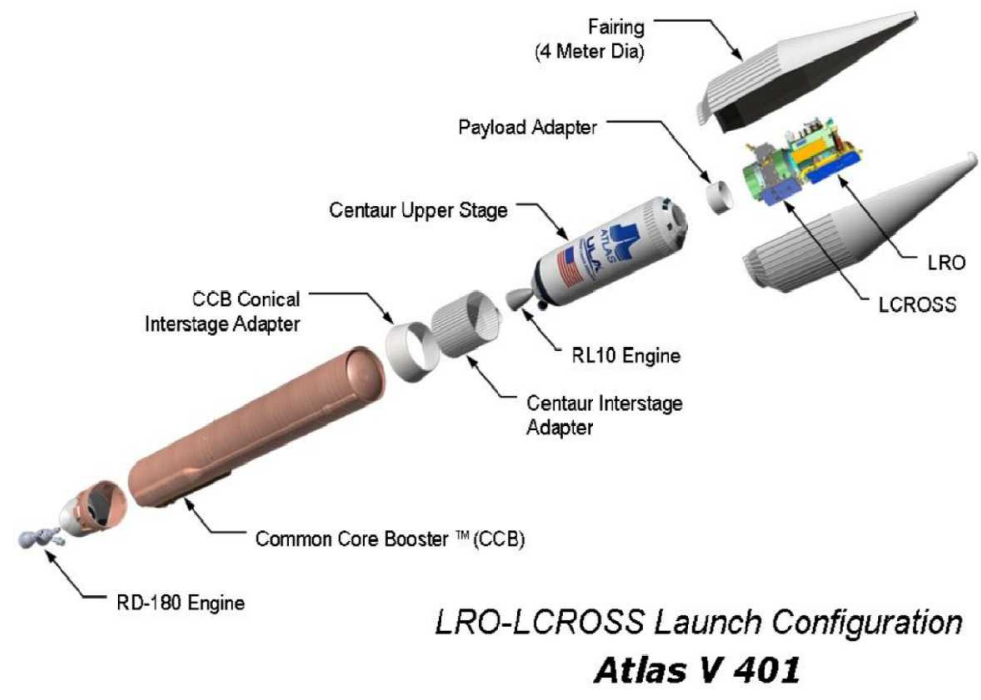

Figure 1. Atlas, Centaur and LRO/LCROSS launch stack commands left LCROSS still under Centaur attitude control. After initial power-up, the Centaur oriented the LCROSS solar array toward the sun to maintain power and thermal control. Then, triggered by the completion of Centaur venting procedures, the Centaur initiated a seventh and final discrete command to signal its release of attitude control to LCROSS. The end of the Centaur's primary mission occurred 32 minutes later, even though it sent beneficial tank telemetry to the ground for an additional 5 hours.

The LCROSS mission was focused on achieving a precise, high-energy Centaur impact within a lunar polar crater, and observing the impact and resulting ejecta plume from the S-S/C and a combination of orbiting and Earthbased assets. To achieve the desired impact geometry, LCROSS performed a lunar gravity assist to transfer from its trans-lunar orbit to a Lunar Gravity Assist Lunar Return Orbit (LGALRO), an elliptical, Earth-centered orbit of roughly lunar semi-major axis, but inclined steeply with respect to the lunar orbit plane (Figure 2). Performing a series of trajectory correction maneuvers (TCM's) with the Centaur impactor still attached, the S-S/C gradually refined the trajectory to hit the targeted crater. Following three revolutions of the Earth, the LCROSS and lunar orbits re-intersected. Less than ten hours before impact, the S-S/C released the Centaur and performed a braking 
maneuver to induce a four-minute delay between the Centaur and S-S/C impacts. This four-minute period allowed the S-S/C to capture and transmit science data from the Centaur impact event before impacting itself and creating a secondary impact plume for possible observation by earth and orbiting resources.

The following project requirements were important factors in guiding spacecraft, launch system and mission operations designs, and are provided here to assist understanding of the following sections.

1) LCROSS will strive to measure the concentration of water in the ejecta cloud, as a ratio of water ice to regolith, to a level as low as $0.5 \%$ by mass, and to determine the form of which the water may be in.

2) LCROSS shall distinguish water carried by the impactor from water present on the moon or provide confidence that any residual water from the impactor is scientifically insignificant for lunar

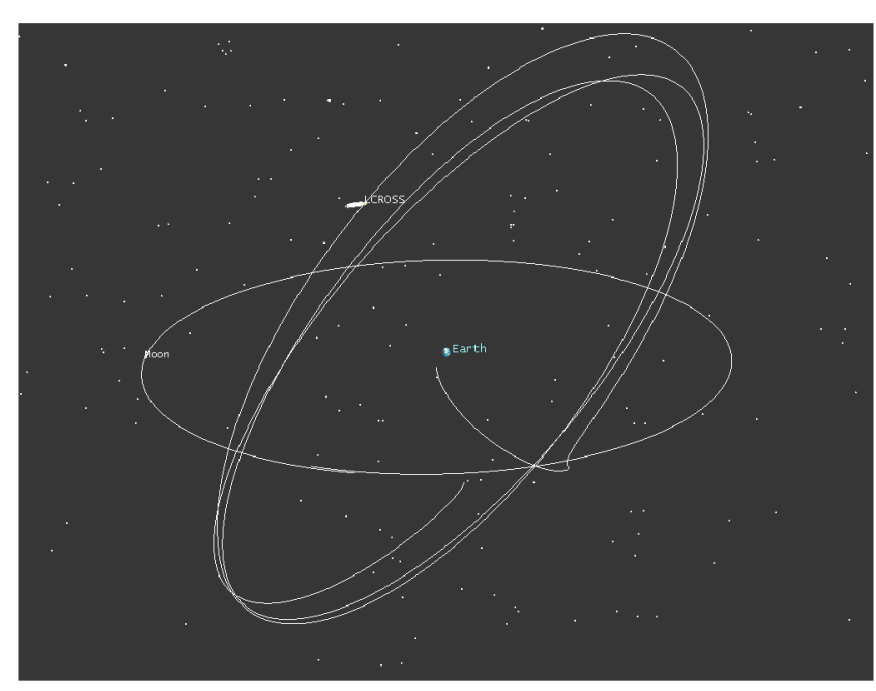

Figure 2. LCROSS flight path and orbit inclination

3) The Launch System shall vent the $\mathrm{H}_{2}$ and $\mathrm{O}_{2}$ tanks to limit the remaining combined mass of those species to no more than $100 \mathrm{~kg}$.

4) The Launch System shall provide the following technical data to support LCROSS design and operations development: a) EDUS Dynamics model; b) Outgassing estimates/reports; c) Venting timeline and accounting of $\mathrm{H}_{2}, \mathrm{O}_{2}, \mathrm{H}_{2} \mathrm{O}, \mathrm{He}, \mathrm{N}_{2} \mathrm{H}_{4}$ remaining in Launch System upper stage lines/tanks; d) Accounting of $\mathrm{H}_{2}, \mathrm{O}_{2}, \mathrm{H}_{2} \mathrm{O}$ contained/absorbed in Launch System upper stage structure/insulation/components.

\section{Centaur Test and Flight Design}

Prior to flight, a number of unique design, analysis, test and flight procedure development activities were required of the launch system provider (ULA) for the project to successfully transport and use the Centaur upper stage on this mission. A typical Centaur mission is on the order of hours, and as the LCROSS/Centaur mission was on the order of 110 days, several risks were identified by the team that were related to the greatly extended mission. Physical modification to the Centaur vehicle consisted of painting the Centaur white for better thermal balance and modeling. Of the numerous requirements placed on ULA by the project, the most significant derived from science contamination constraints placed on the mission. As described in the previous section, requirements were developed to eliminate contamination of the lunar impact site by the impactor itself. ULA analyses and tests were performed to better understand Centaur propellant valve performance, tank depletion rates, and to estimate the quantity of water that might be entrained in the Centaur insulating foam after liftoff. These included Centaur Tank Pressure Control Analysis, which was a thermal control analysis to provide maximum predicted steady-state temperature of vapor mass in the tank after LCROSS handoff. ULA analysis also estimated that approximately $1 \mathrm{~kg}$ of water may have been trapped in the Centaur foam, half of which could be boiled off when the Centaur cold-side was exposed to the Sun. The other half would be baked out during normal mission cruise (solar panel side exposed to sun). Per ULA analysis, $90 \%$ of this trapped water would bake out within one hour of sun exposure. Another unique analysis and set of tests were conducted to quantify the total weight of water entrained in the paint on the Centaur. Results confirmed low water mass in the paint. Unique ULA flight software product development included a Centaur propellant tank blowdown (post-blowdown sequence), designed to ensure $\mathrm{O}_{2}, \mathrm{H}_{2}$ and helium pressure reduction for minimal venting after LCROSS handoff. An additional Solenoid Vent Valve operation was added late in the design to improve the amount of gas expelled during this procedure. Software adjustments and tests were performed on this Centaur propellant tank blowdown sequence to additionally balance tank pressurization and venting, and again minimize post handoff venting. Onboard Centaur processing controlled all Centaur maneuvering, initiated the LRO separation, and issued discrete commands that triggered LCROSS activation. The following is a list of designed nominal propellant depletion maneuvers for both the cryogenic main engine propellants and pressurants, and 
hydrazine-based reaction control systems (RCS). Actual event timing differed as some events were activity driven. Launch relative timing and locations of LCROSS discrete signals are included as a reference:

1) Attitude change and impulsive blow down ( $L+\sim 61$ minutes, continuing to $L+73$ minutes).

2) Non impulsive spin for RCS Hydrazine depletion burn for $\sim 46$ minutes ( $\mathrm{L}+\sim 77$ minutes).

3) Hydrazine non-impulsive burn off for $\sim 7$ minutes ( $\mathrm{L}+\sim 110$ minutes).

4) Guided main engine hydrazine burn, $(\mathrm{L}+\sim 126$ minutes).

5) Issue LCROSS discrete signals \#1 - \#6 ( $\mathrm{L}+\sim 132$ minutes).

6) Hydrazine final depletion, ( $\mathrm{L}+\sim 189$ minutes).

7) Issue LCROSS discrete signal \#7 to handoff control, (at Hydrazine depletion).

After launch, Centaur 'end of mission' and control handoff to LCROSS, ULA engineering teams continued monitoring Centaur tank pressure and temperature telemetry for five hours beyond what would be considered for a normal launch. These additional data were used to refine estimates of propellant depletion and to predict subsequent residual valve leakage, important to the prediction of LCROSS propellant used to maintain attitude control (see next section). One final analysis item the ULA team addressed was structural dynamics of an empty Centaur. The concern was that Centaur structure, being empty after blowdown, could flex during TCMs, affecting the accuracy of those burns. Analysis was performed for an empty Centaur to show that the structure was rigid enough to survive and not affect planned maneuvers.

Whereas a good discussion of these and other efforts performed by ULA, and not mentioned here, is beyond the scope of this paper, it should be noted that many of the efforts to accommodate LCROSS mission requirements from the launch provider perspective were unique and significant. Future projects of this nature are advised to budget sufficient time and resources to conduct early and frequent interactions with the launch vehicle provider to define and mitigate unique and unexpected requirements that may never have been imposed on previous missions.

\section{Spacecraft Maneuver and Attitude Control Design}

Transporting and then releasing the Centaur upper stage as part of the LCROSS mission created unique spacecraft attitude control system (ACS) design challenges. Two distinct ACS gain sets were required, one set for mass properties of an attached Centaur configuration (most of mission), and one for operating the S-S/C alone following Centaur separation (for the last 9.5 hours of the mission). It should be noted that this was not simply a matter of designing two different controller gains. The required effort is similar to designing controllers for two different spacecraft because it entails the gain synthesis, stability analysis, simulations and flight software development for cruise, maneuvering, delta-V, pointing, and safe modes for two drastically different configurations. The more than a factor of 5 reduction in mass, nearly a 4 meter shift in the longitudinal location of the center of mass, and approximately a factor of 200 reduction in transverse moments of inertia without attendant change in thruster size or locations delineate the configuration challenges that had to be addressed and which were solved by judicious thruster placement and alignment, and careful propellant budgeting. Also, the mission operations team had to provide and test commanding products, prepare contingency procedures, and run simulations of operationallyrelevant scenarios with various combinations of both gain sets. Impacting this process was a question of whether the Centaur c.g. could be affected by the success of the blowdown. This question and the fact that the Centaur was nearly symmetric about the LCROSS $\mathrm{Y}$ and $\mathrm{Z}$ coordinates, caused an overlap in $\mathrm{Y}$ and $\mathrm{Z}$ c.g. error estimates, making it difficult to know which axis was the principal one. This was important for selected contingency analysis.

Creative solutions to configuration-related problems were called for and whereas their detailed description is beyond the scope of this paper, it is illustrative to mention two examples. The first was the problem of the large sun sensor blind spot created by the attached Centaur. This problem was circumvented by orienting the S-S/C's coarse sun sensors and modifying their processing algorithms to assure that, as part of a safe mode contingency, the combined vehicle could find and point its solar array at the sun from any attitude. The second arose in considering the operations and contingencies for Centaur separation. The high control-authority gains used when the Centaur was attached had the potential to drive the S-S/C unstable if used when detached. Alternatively, premature transition to S-S/C only gains without successful separation may have led to sluggish attitude control because controller gains for the separated configuration would have been insufficient to hold the attitude of the unseparated vehicle. Careful designs, numerous simulations, unhindered communications between Northrop Grumman and NASA teams, and many hours of rehearsals were ultimately the tools for finding creative solutions to these challenges. Future projects incorporating separation events with significant mass properties changes during the mission should anticipate the amount of design and operations effort required. 


\section{Centaur Venting}

Analysis of Centaur components provided by ULA before launch exposed a potential for residual propellants to leak from one or more valves on the spent stage for weeks or even months following launch. Even after the Centaur performed propellant depletion activities in the first few hours after launch, the propellant control valve states could not be determined or controlled and hence any residual fuel remaining could vent and induce torques on the S-S/C and Centaur system. This generated a significant risk for LCROSS. Whereas these valves perform well for the launch and orbit maneuver activities, they were not designed for $100 \%$ closure after the Centaur's task is completed (not a system requirement and not previously needed). Reliably predicting the amount of S-S/C propellant needed to counteract venting disturbance was difficult because of the uncertainty in the magnitude of the leak rate, the direction that the escaping gasses would take (depending on number of valves open, their location, and effective thrust parameters including leak vector), and the decay time associated with such a leak. Because of these uncertainties, in the weeks immediately prior to launch, the analysis proceeded to bound the problem by considering four constant leak rates with a direction chosen to produce disturbance torques predominantly about the LCROSS Xbody axis. In this direction, the LCROSS attitude control thrusters had the least control authority and would therefore expend the largest amount of propellant to counteract the Centaur venting. The predicted severity of this effect depended on expected leak rate. Among the admissible set of leak rates, the low range at $800 \mathrm{sccm}$ (Standard Cubic Centimeter per Minute) represented the most severe consequences because analysis suggested this type of leak would cause a moderate disturbance torque that would persist for much of the mission. Under slow and moderate leak rates, analysis showed that the nominal control mode designed for the cruise portion of the mission (lasting on the order of 100 days) would consume all the propellant ( $305.5 \mathrm{~kg}$ launch load) before completing the mission. Very high leak rates would empty the Centaur tanks more quickly, and hence have a smaller overall effect. Note in all cases, the assessment was for the worst-case set of Centaur valves (the valves causing torques about the $\mathrm{X}$-axis). Leaks from different valves would yield less severe results)

The LCROSS project developed a number of operational strategies for assessing leak severity and two alternate modes of operating the vehicle to counteract leak effects. Through analysis and simulations, it was shown that adapting the cruise control gains to work with increased thruster pulse width $(50 \mathrm{~ms}$ to $100 \mathrm{~ms})$, which increased the low duty cycle efficiency of the pulses, and by changing the thruster selection logic to fire thruster pairs instead of quads, a factor of 2 savings could be realized in counteracting the leak disturbance. Although a considerable improvement, this higher efficiency stellar inertial mode by itself was not considered sufficient to preserve the mission if leak rates greater than $800 \mathrm{sccm}$ were encountered early on. To provide mitigation under such circumstances another approach was investigated, which entailed spinning the vehicle about its axis of maximum inertia with the solar array (vehicle $-Y$ body axis) pointed at the sun. This high efficiency sun pointed mode relied on the dynamic stiffening and disturbance torque averaging offered by spinning the vehicle about its maximum principle axis and using the thrusters only to maintain the spin rate. Its application in flight, however, remained dubious because of unfavorable LCROSS mass properties. From the as-built mass properties estimates, at control handoff the LCROSS Y-axis moment of inertia was estimated as 74,330.2 (+/- 226.4) $\mathrm{kg}-\mathrm{m}^{\wedge} 2$, whereas the Z-axis inertia was estimated as 73,996.1 (+/-226.4) $\mathrm{kg}-\mathrm{m}^{\wedge} 2$. Even with the most favorable allocation of the uncertainties, the $\mathrm{Y}$-axis was therefore the major axis of inertia by only $1.1 \%$. This margin was too small given the attendant fuel slosh, structural flexing, etc., to assure that the spin condition once induced on the vehicle would preserve a spin about the desired axis and assure a power and thermally safe configuration. LCROSS launched with this risk, but with a reasonable expectation that actual Centaur leak behavior would be far less than predicted by worst-case analyses. It should be noted that these efforts expended significant resources from many groups at a time when launch preparations and operations tests were in progress. Furthermore, at the LPRP program level, the risk posed by potential Centaur leak rates influenced the decision to slip the LRO/LCROSS launch from the June 2 launch block (with high delta-v requirement, and hence low propellant margin) to the June 17 launch block (higher propellant margin; included 6/17, 18 \& 19). Under expected leak conditions, and with the above strategies available, LCROSS showed a very good probability of torque mitigation and propellant margin for the June 17 launch window.

Hours into flight, the mission operations team assessed the severity of Centaur leaks using three methods. It performed a series of disturbance torque assessments by observing the spacecraft attitude with control disabled; observed the deadbanding behavior while under attitude control; and measured propellant usage due to active attitude control. These proved invaluable in characterizing the magnitude, direction, and temporal characteristics of the net torque. Figure 3 shows the results from torque observations collected 19.5 and 26.5 hours after launch with thruster control inactive. Comparing the two, the yaw torque values decreased by $35 \%$, and roll torques diminished to nearly zero. Mission operations concluded within the first 2 days of the mission that remaining Centaur gasses 
were inducing only a moderate torque on the combined stack, and since the estimates of the net disturbance tracked closely with the estimated propellant consumption rate, it was evident it was evident that propellant use to counteract the propellant venting was not going to jeopardize the mission.

LCROSS

exhibited a peak propellant usage of approximately $6.5 \mathrm{~kg} /$ day while in cruise at the beginning of the mission, a factor of about 10 above the predicted nonleak value. This usage ramped down quickly as
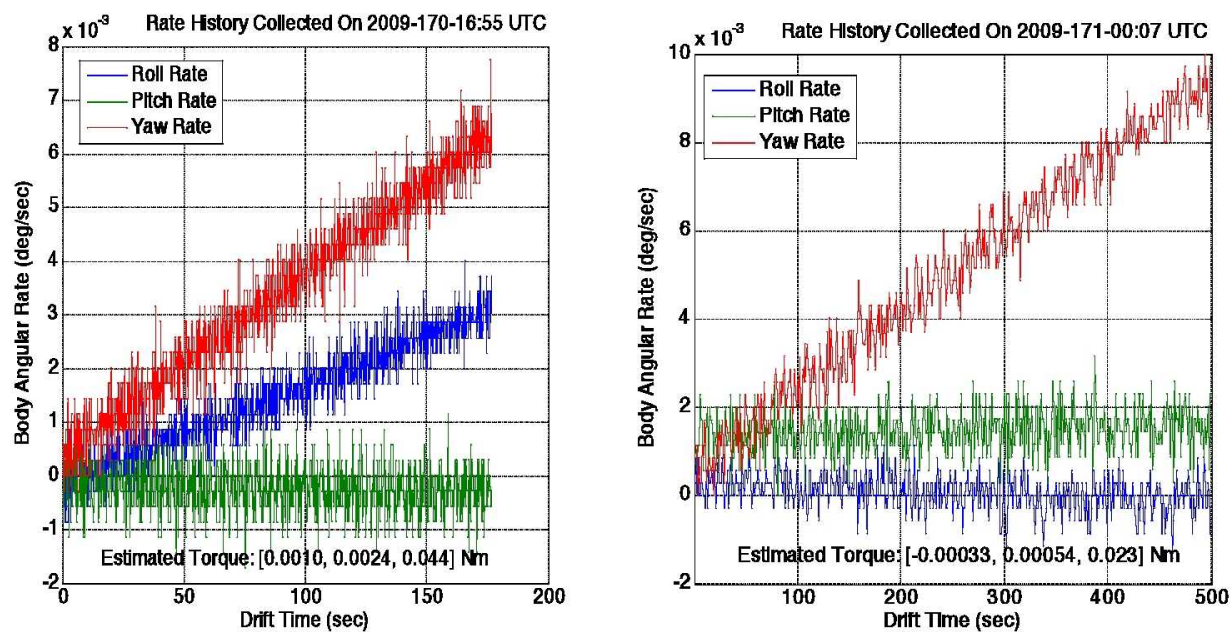

Figure 3. Torque assessments of Centaur leak with S-S/C ACS off Estimates taken during drift (note different durations plotted) at 19.5 and 26.5 hours after launch the disturbance torque decayed rapidly the first couple of days. Within a few days the usage rate was $<1 \mathrm{~kg} / \mathrm{day}$. By the end of mission, propellant usage in cruise had shrunk to $0.26 \mathrm{~kg} / \mathrm{day}$. To minimize propellant usage, the team configured the spacecraft to operate with paired thrusters instead of nominal quads. Whereas this helped with fuel usage, it also removed the cancellation effect of the balanced four-thruster sets, causing all ACS firings to perturb the LCROSS orbit slightly. The team also increased the ACS impulse bit from $50 \mathrm{~ms}$ to $100 \mathrm{~ms}$, thereby improving the effective specific impulse for typically short thruster firings inherent to cruise mode. Despite the significant increase in propellant usage compared to a no leak case, the propellant margin associated with the June 18 launch date was sufficient to make this usage tolerable.
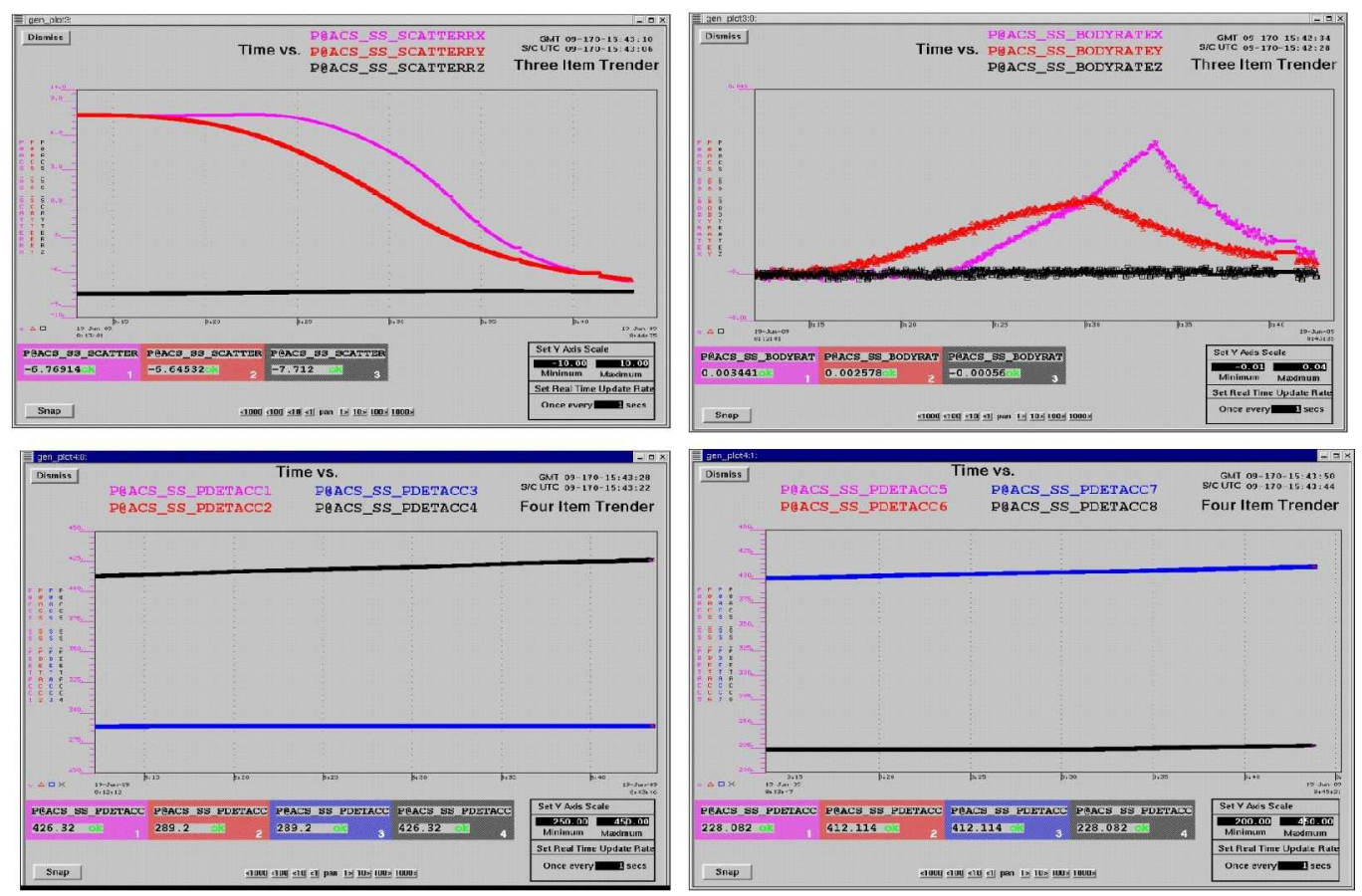

Figure 4. Unexpected Torque changes recorded during cruise

3-axis body rate and attitude error (upper) and 8 thruster outputs (below) all versus time.

\section{6}

American Institute of Aeronautics and Astronautics
Another behavior potentially associated with venting during flight, was the apparent occasional change in direction of the disturbance torques, which occurred in discrete jumps.

Because these effects were only indirectly observed through rate 
behavior about each axis, it was never determined if Centaur venting was the cause, or whether there were other effects in play (such as ice chunks separating from the Centaur). The plots in figure 4 illustrate a sudden and pronounced change in the attitude deadbanding behavior (3-axis attitude \& body rate plots on top) without appreciable change in the accumulated thruster pulses (bottom plots). Numerous such events were observed in the early portion of the mission and continued to occur on a less pronounced scale well after the first five days.

A major lesson learned from the Centaur venting analysis and mitigation process is when flying systems not designed for extended use, or using launch components well after their design lifetimes, expect to spend extra effort in the discovery, analysis and planning for newly discovered behaviors. Project teams must investigate all potential behaviors of the system to be launched and flown to anticipate problem behavior and to develop strategies to counteract them (via analysis, risk assessment, and operational mitigation strategies). Equally important for this investigation process will be to find and use potentially advantageous and mission beneficial behaviors and design operations to take advantage of them.

\section{In Flight Cold Side Bakeout}

Water imbedded in the Centaur insulating foam or attached to the surface was a known threat for both science contamination of the impact site as well as final impact targeting. After S-S/C separation, any significant amount of water remaining on the Centaur (even if within science requirements) had the capability to significantly alter the Centaur course. As the Centaur tumbled or rolled prior to impact with no S-S/C control, it would expose its 'cold side' to the sun, and subsequent water outgassing would impart additional vectored thrust, throwing it off target. The mission operations team mitigation strategy was a pre-emptive and controlled Centaur outgassing maneuver, performed during the cruise phase of the mission, termed Cold Side Bakeout (CSB). The objective of the maneuver was to expose the cold, anti-sun side of the Centaur temporarily to the sun with the objective of removing water on the surface and in the foam. The secondary objective was to either verify no further outgassing was present, or, estimate the quantity of water remaining. Since escaping water was predicted to cause small spacecraft velocity changes, precise Doppler measurements taken during CSB maneuvers could also be used to assess whether further out gassing was needed. As previously noted, ULA estimated up to $1 \mathrm{~kg}$ of water may have been embedded in Centaur foam. One CSB was originally planned, (although planning for subsequent bakes was to be a function of results from the first). Three CSB's were actually executed. This resulted in fair, but not complete certainty that sufficient water had been removed from all portions of the Centaur. These tasks were added to the list of required mission events and while not a contributor to mission duration, they did add to operations planning, product development and testing.

Prior to CSB, there were two predictions on CSB effectiveness - one expected virtually no effect of the maneuvers, under the theory that water would be deeply entrained in the Centaur foam, and that short exposures of one hour or less would not be sufficient to melt and/or sublimate the water out of the matrix. The second predicted a noticeable response, perceptible in spacecraft dynamics in terms of torque and delta-v. Per report from Beagle-2 failure review board, it was postulated that the sublimation of 50 grams of ice could perturb a 1 ton spacecraft by 2.5 $\mathrm{mm} / \mathrm{s}$ in the anti-sun direction $^{5}$. It was expected that LCROSS

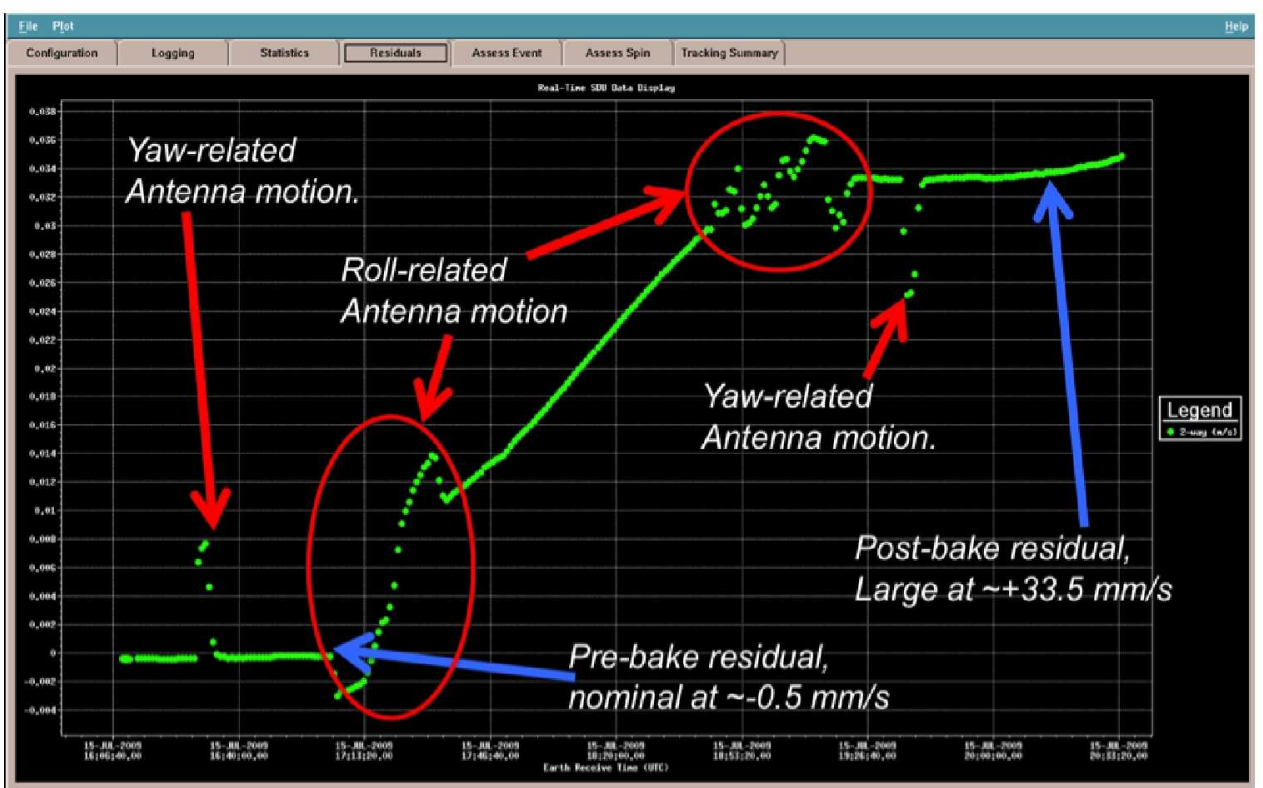

Figure 5. Doppler results from Cold Side Bake \#1

Vertical units are in $\mathrm{m} / \mathrm{s}$, where 1 tick is $2 \mathrm{~mm} / \mathrm{s}$. The actual bake event occurred over 90 minutes, and total shift in line-of-sight Doppler was $33.5 \mathrm{~mm} / \mathrm{s}$. 
might see a total perturbation of $\sim 1 \mathrm{~cm} / \mathrm{s}$, with the acceleration dramatically tapering off towards end of bake.

\section{A. Cold Side Bake No.1}

The execution of the first CSB\#1 (on DOY 196) rolled the S-S/C \& Centaur assembly 180 degrees over 40 minutes, dwelled 60 minutes, and then rolled back to cruise attitude over another 40 minutes to 'bake' out water on the 'back side'. The primary indication of water outgassing while in CSB and success of the maneuvers upon completion were changes in spacecraft velocity, derived from Deep Space Network (DSN) Doppler residuals provided by the Navigation team. CSB \#1 results indicated a $3.4 \mathrm{~cm} / \mathrm{s}$ change in velocity, correlating directly with the bakeout period (see fig. 5), three times higher than expected. The initial slightly higher rate, may have been a legitimate change in the outgassing characteristic, or a subtle effect due to the change in direction of the outgassing vector as the spacecraft rotated. Also, the level of acceleration did not dissipate over the course of the dwell period, indicating there was likely more water to be released from the Centaur at maneuver completion. The team noticed

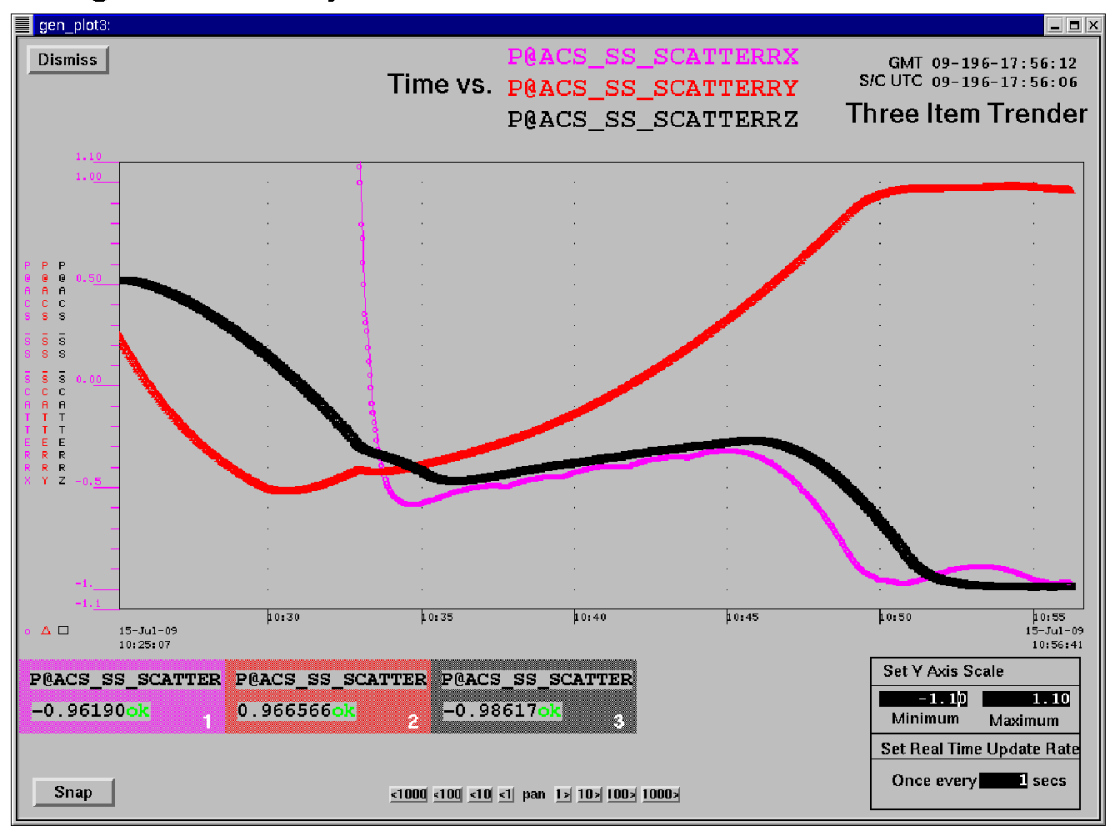

Figure 6. Attitude Error during Dwell in Cold Side Bakeout \#1

Three-axis attitude error vs. time showing sticking to deadbands (+/-1.0 deg.) torques about the $\mathrm{X}$ and $\mathrm{Z}$ axes (as evidenced by sticky deadbanding) over the same time period with no appreciable change in these torques while anti-sun pointing (see fig. 6). Transients in the acceleration seemed brief; it did not take much time for the cold-side to "warm-up" before seeing an effect on the trajectory, and it did not take much time for the acceleration to diminish once the vehicle's cold side was returned to shadow. Based on these observations, targeting errors anticipated for the Centaur under similar rates of outgassing at impact were estimated to be one to two kilometers. This was based on three assumptions: 1) the cold-side of the Centaur would be exposed to sunlight for half of the time between separation and impact ( $\sim 4.5$ hours), 2$)$ the separated Centaur was $\sim 30 \%$ lighter than the mated flight system, causing accelerations to be larger, and 3) the cold-side material would not appreciably ablate in that time period, and that no beneficial "cancellation" of perturbation would occur due to fast acceleration transients. Propellant usage for the first cold-side bake was estimated to be 0.6 to $0.9 \mathrm{~kg}$.

Another result of the first CSB was suspected, but possibly not fully appreciated. On the outbound slew to bakeout attitude, a star tracker (STA) error tripped ACS fault protection and transitioned the STA to Standby Mode. The operations team postulated that the most probable reason for this event was either water outgassing and obscuring the STA field of view, or sunlight glinting off of the STA sunshade. A much brighter background would then result causing an appreciable increase in the STA background count level leading to the STA faulting into standby mode. No confirmation of these suspected causes was possible, but an increase in STA background count did re-occur to a lesser degree in each of the next two cold bakes. Two attempts at STA recovery failed while in bake roll attitude, and telemetry observation of background noise confirmed high levels ( $>1200$ instrument counts), indicating continued interference. These levels returned to normal ( 820 counts) after rolling back to cruise attitude at the end of the first CSB.

\section{B. Cold Side Bake No.2}

Cold Side Bake \#2 maneuver was executed on DOY 211 without incident. It consisted of a 10 minute, 180 degree roll to bake attitude, 100 minutes in bake, and another 10 minute slew back to cruise attitude. Based on a continuation of outgassing at the same level as CSB\#1, a hypothetical delta-v for CSB\#2 of 5-6 cm/s was estimated. After accounting for look-in angles between the Earth line of sight and the spacecraft anti-sun vector, the actual hypothetical delta-v was $\sim 1.13 \mathrm{~cm} / \mathrm{s}$. Navigation reported that only about $20 \%$ of the expected delta-v was observed 
based on Doppler results, which implied that a knee in the outgassing effects was passed meaning a very effective first bake and/or additional cook-off of cold-side material occurred for hours or days after Cold Side Bake\#1. As a result, a third bakeout was designed to orient some intermediate surfaces of the Centaur to the sun and confirm effectiveness of the first two CSB's. The STA background noise level grew from the nominal 820 counts to about 980 counts and then slowly decreased while in bake attitude. Levels returned to normal at completion of the maneuver. Assessed propellant usage for the second cold-side bake was 1.6 to $1.7 \mathrm{~kg}$.

\section{Cold Side Bake No.3}

The third bakeout was successfully performed on DOY 267 in conjunction with an antenna test. The maneuver this time performed a slow roll to 135 degrees and dwelled 20 minutes, then rolled to 225 degrees and dwelled another 20 minutes, and finally rolled the final $135 \mathrm{deg}$. back to cruise attitude. Total time for execution was 125 minutes. This allowed the full exterior of the Centaur to be exposed to sunlight for a number of minutes, with the potential to induce sublimation in areas where previous CSB's had not.

Based on results from CSB\#2, the hypothetical delta- $\mathrm{V}$ in the antiSun direction was estimated to be $7.1 \mathrm{~mm} / \mathrm{s}$ for the 1 hour and 15 minutes spent within 45 degrees of the sun. Due to the $81^{\circ}$ sun-probeearth angle at the time of the activity, only $14 \%$ of the delta-V would be seen in the Doppler shift, equivalent to $1.0 \mathrm{~mm} / \mathrm{s}$. Due to the attitude changes midway through the activity, observation of the offgassing delta-V during those would be obscured by Doppler effects due to antenna motion and velocity changes due to thruster firings. The Navigation team reported effects from this third bakeout were at much smaller levels than experienced with CSB \#1 and similar to CSB \#2. Thruster

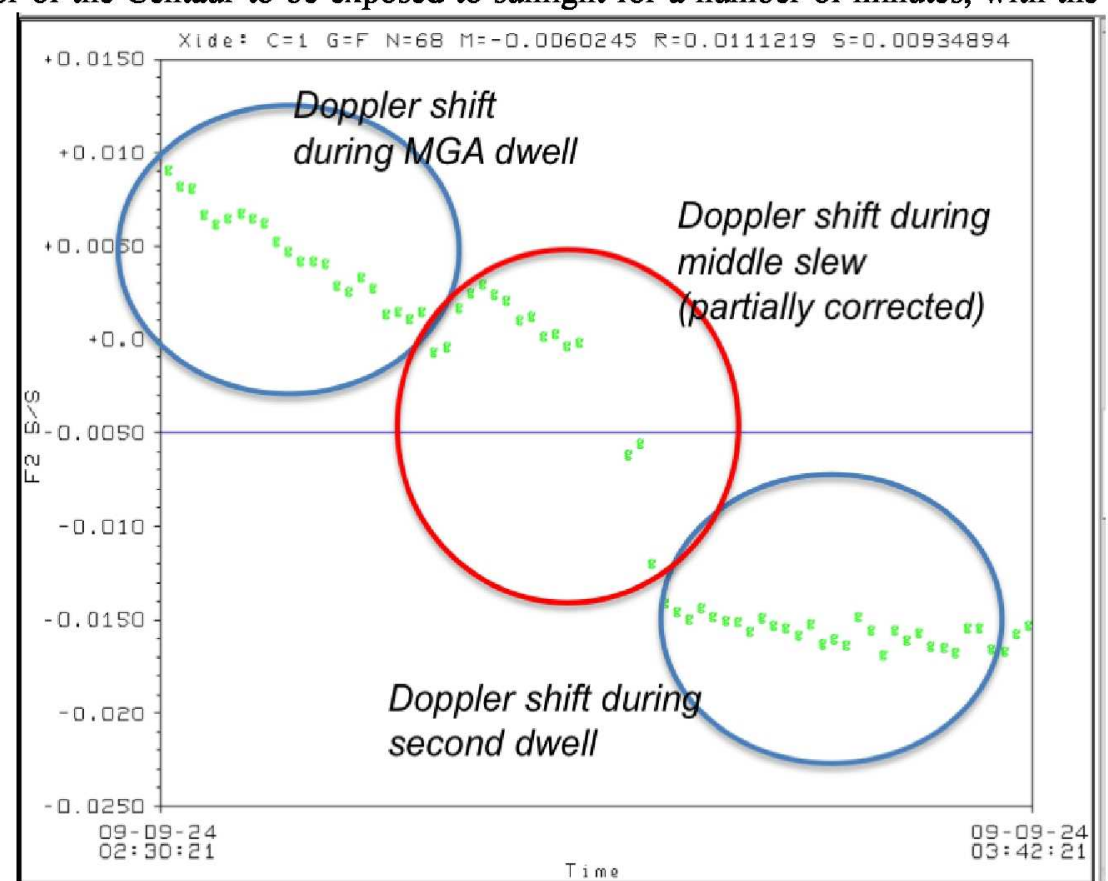

Figure 7. Processed Doppler effects from Cold Side Bake \#3 DOY 267 (Doppler residual freq. vs. time) perturbations effects however were on the order of 0.5 to $1.0 \mathrm{~mm} / \mathrm{s}$, which inhibited establishing a longer-term outgassing trend for this activity. The results (Figure 7) did provide increased confidence in the CSB\#2 targeting projections. Total delta-V measured was $1.2 \mathrm{~mm}$ (with partials of $0.45 \mathrm{~mm} / \mathrm{s}$ observed while rolled $135^{\circ}$, and $0.13 \mathrm{~mm} / \mathrm{s}$ observed while rolled $225^{\circ}$ ), which also tapered off well towards the end of the maneuver. If the delta-V was anti-Sun, as hypothesized, then the outgassing acceleration during CSB\#3 dwell was $9.2 \mathrm{~mm} / \mathrm{s} / \mathrm{hr}$. This was larger than the acceleration observed during CSB\#2, and implied that the outgassing delta-V may have been asymmetrical, rather than purely anti-Sun. This implication was borne out by a $70 \%$ reduction in rate of Doppler shift seen during the second dwell, after the spacecraft had been rotated $90^{\circ}$. The slopes in the Doppler shift during each dwell were relatively flat, implying a floor in the outgassing performance had potentially been reached. Assuming these perturbations to be applicable for half of the post-separation time $(9.7$ hours), the total delta- $V$ would be $3.7 \mathrm{~cm} / \mathrm{s}$. For this delta- $\mathrm{V}$, total deflection at lunar crater target was estimated at 640 meters, and after applying cosine losses to accelerations during Centaur rotation, the impact deflection was then bounded to be 410 meters. Whereas this was well within the targeting requirement, a nominal biasing of impact target based on sun location was proposed and applied by the Mission and Maneuver Design team, with input from the Navigation team. For this maneuver, the STA background noise levels grew to 852 counts with no STA fault and returned to nominal at maneuver completion. Assessed propellant usage for CSB \#3 was estimated at $0.47 \mathrm{~kg}$. Monitoring accumulations of thruster firing 9 hours later, the thruster firing totals were about $1 / 3$ of what had been occurring, implying that the leakage or off-gassing had gone down noticeably over the course of the maneuver. Given that the Doppler data from CSB \#3 was acquired at a less than ideal angle, 
and indications were that significant outgassing was still possible, the team desired to perform one more cold side bake. However, due to a previous S-S/C issue there was not enough fuel or time remaining to make another attempt.

Results from these Cold Bake maneuvers validated pre-launch concerns and indicated successful depletion of remaining moisture from the Centaur vehicle. It is also worth noting that the larger than-expected perturbation observed during CSB\#1 may be an indication that much more then one $\mathrm{kg}$ of ice may have been carried to orbit on the upper stage, which correlates with ULAs predictions of water ice trapped in the foam $(\sim 1 \mathrm{~kg})$ plus additional water ice attached to the surface (more than one $\mathrm{kg}$ ). These maneuvers also required significant effort from and exercised all components of the mission operations team. Pre planning for these events was therefore crucial in the mission operations planning phases.

\section{Thermal Design}

Carrying the Centaur for this mission duration complicated pre-launch thermal analysis and in-flight thermal control of the LCROSS S-S/C. Five factors contributed:, 1) the thermal sink of the large mass, 2) radiative properties of the Centaur, 3) reflective properties of the Centaur, 4) large potential sun shadow created by the Centaur, and 5) coatings or surface preparations that could potentially flake or have thermal property changes. Unique concerns included the thermal characterization of the Centaur's mating electrical connector capability, life and function of the separation clampband and potential leak or burst hazard of Centaur batteries while in proximity to the S-S/C solar array. Note that LCROSS initiated separation of S-S/C and Centaur via the Centaur clampband, which is normally initiated by the Centaur. Additionally, analysis was performed on the Centaur-payload adapters to identify the best coatings for the expected thermal environment. While these warrant additional thermal analysis, the LCROSS design was limited to what could be afforded under project Class D budget constraints.

The thermal design needed to accommodate these factors with the Centaur attached to S$\mathrm{S} / \mathrm{C}$ (normal mission analysis), yet still needed to satisfy thermal constraints when the Centaur was detached for the critical last 9+ hours of the mission. LCROSS suffered from a thermal control problem that affected some of its 5 $\mathrm{N}$ thrusters ${ }^{4}$. Whereas the cause of this anomaly was not related to the Centaur,

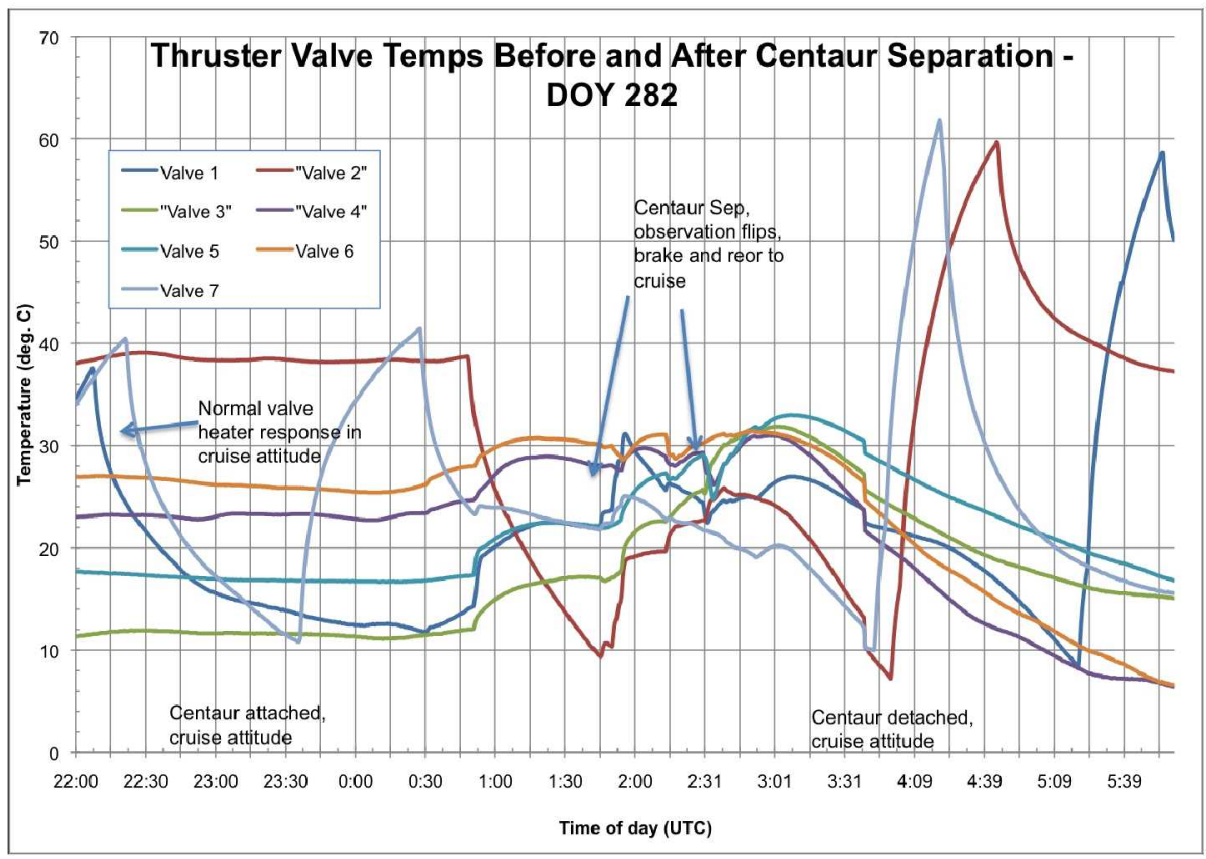

Figure 8. Thruster valve temperatures before and after Centaur detach

the Centaur's proximity to the affected thrusters was expected to influence the thruster valve temp response. This increased the uncertainty of how the valve temps would respond once the Centaur was released. Hence, there was a potential operational risk during a critical portion of the mission if further mitigation actions were to be required. Figure 8 shows S-S/C thruster valve temperatures both before and after Centaur separation indicating the change in thermal behavior observed. These behaviors did not impact LCROSS operations, but the level of uncertainty about these changes warrants diligence on future projects.

\section{Centaur Separation}

Operation of the Centaur release mechanism required additional pre-launch analysis and mission operations design, planning and testing. The Centaur Payload (spacecraft) Release System is designed to separate the spacecraft from the Centaur as soon as orbit insertion maneuvers have been completed, typically within 1-2 hours of launch. 
The LCROSS project required that the S-S/C and Centaur remain attached, and for this release mechanism to function properly while in cold space after 112 days. This had never before been attempted with this launch system, and although an analysis showed that the mechanism would still function, there was no certainty. Significant analysis was spent on establishing the thermal environment at the structure and connectors that needed to disconnect properly when the Centaur was released. The resulting uncertainty required an operational separation contingency plan to retry separation from the ground, or fly the mission to completion with the Centaur attached. The contingency response was designed based on the assumption that the release pins or mechanism might be frozen (where not exposed to sunlight), and that a roll to expose the cold side (as was done with the Cold Side Bakes) to the sun might warm the release mechanism sufficiently for a second release attempt to be successful. At the time this contingency would have to be executed, mission operations would have already transitioned the ACS to 'detached' rates in anticipation of a successful separation. The following steps outline the additional mission planning activities that needed to be anticipated, created and tested prior to flight for this contingency.

1) ACS software flag reset to 'attached' (control algorithms based on mass).

2) Stop currently running on board sequence (which assumes successful first separation attempt).

3) Command a new set of quaternions to roll cold side of release mechanism into sun.

4) Roll back to separation attitude and re-send release commands

5) Re-enter nominal procedural step to observe separation attempt results.

6) Time and event planning so that these events could be performed with enough time remaining to accomplish remainder of mission.

Had both separation attempts failed, mission objectives dictated that the S-S/C and Centaur would impact the target crater still attached. Even as this may have created a larger impact plume for other observation assets due to the increased mass of the LCROSS S/C, a significant mission objective would be lost as the onboard science instruments would not be able to observe the impact. Additionally in this failure mode, there was risk of impact science measurement contamination due to unused fuel aboard the LCROSS S-S/C now added to the impact site.

\section{Conclusions}

The LCROSS spacecraft launched with the Lunar Reconnaissance Orbiter on June 18, 2009, and on October 9, 2009, after 112 days in flight, the LCROSS S-S/C successfully delivered both the Centaur and itself on-target as kinetic impactors to the lunar crater Cabeus. The LCROSS project designed, launched with and carried the Centaur upper stage vehicle for the duration its mission, and experienced unique behaviors associated with that union. Nearly every phase of this project was impacted by these shepherding considerations. This mission demonstrated one successful use of a Centaur upper stage as an integral part of a science mission and how many of the unique challenges in designing and flying such components can be overcome. Future missions will likely find enabling uses for spent launch vehicle components for cost reduction or as payloads. We expect that some of the specific lessons learned on LCROSS will also apply to these missions, and that the general approach of early and sustained close coordination with the launch vehicle provider will be necessary to ensure mission success.

Appendix A

Acronym List

\begin{tabular}{|l||l||l||l||}
\hline ACS & Attitude Control System & LRO & Lunar Reconnaissance Orbiter \\
\hline CSB & Cold Side Bakeout & MGA & medium gain antenna \\
\hline ConOps & Concept of Operations & RCS & Reaction Control System \\
\hline DOY & day of year & S-S/C & Shepherding Spacecraft \\
\hline DSN & Deep Space Network & STA & star tracker assembly \\
\hline EDUS & Earth Departure Upper State & TCM & LCROSS Trajectory Correction Maneuver \\
\hline EELV & Evolved Expendable Launch Vehicle & TLI & Trans-Lunar Injection \\
\hline ESPA & EELV Secondary Payload Adaptor & ULA & United Launch Alliance \\
\hline LCROSS & Lunar Crater Observation and Sensing Satellite & & \\
\hline LGALRO & Lunar Gravity Assist Lunar Return Orbit & & \\
\hline LPRP & Lunar Precursor Robotic Program & & \\
\hline
\end{tabular}

11

American Institute of Aeronautics and Astronautics 


\section{Appendix B \\ Glossary}

\begin{tabular}{|l||l||}
\hline Centaur upper stage & Second stage of the Atlas launch vehicle. \\
\hline \hline Cold-side bakeout & Maneuver to expose previously shadowed surfaces to the sun to cook off volatiles \\
\hline \hline Lunar impactor & $\begin{array}{l}\text { Vehicle, device or mass used to impact the lunar surface for the purpose of exposing } \\
\text { buried material. }\end{array}$ \\
\hline \hline Science data contamination & Invalidation of science return do to addition of compromising or diluting materials \\
\hline \hline Sticky Deadbanding & Vehicle attitude observed to drift or move to and stay at attitude deadband limit \\
\hline \hline Venting unused propellants & Release and clearing of any unburned launch or control system fuels. \\
\hline Water outgassing & Release or sublimation of water ice attached to or entrained in component surfaces.| \\
\hline
\end{tabular}

\section{Acknowledgments}

This work was funded wholly by NASA under the LCROSS project.

The authors wish to thank Dirk Schreier, United Launch Alliance for content and review of ULA, Atlas and Centaur related text.

\section{References}

${ }^{1}$ Goodwin, J. S. and P. Wegner, "Evolved Expendable Launch Vehicle Secondary Payload Adapter: A New Delivery System for Small Satellites," Proceedings of the Fifteenth Annual Small Satellite Conference, Logan, UT, August 2001

${ }^{2}$ A. Lo, H. Eller, D. Dailey, E. Drucker, J. Wehner, "Secondary Payloads Using the LCROSS Architecture", AIAA Space 2008 Conference, Vol. TBD, American Institute of Aeronautics and Astronautics, Washington, DC, 2008, pp. TBD-TBD.

${ }^{3}$ Secondary Payload Planner's Guide For Use on the EELVS Secondary Payload Adaptor, Space Test Program Office, Space and Missile Systems, Kirtland AFB, NM. 2005

${ }^{4}$ P. Tompkins, et al., "Flight Operations for the LCROSS Lunar Impactor Mission", AIAA Space Ops 2010 Conference, Delivering on the Dream, American Institute of Aeronautics and Astronautics, Washington, DC, 2010, Paper 2010-1986 (submitted for publication)

${ }^{5}$ Beagle 2 ESA/UK Commission of Inquiry, R. Bonnefoy, Chairman, April 2004, page 37. 


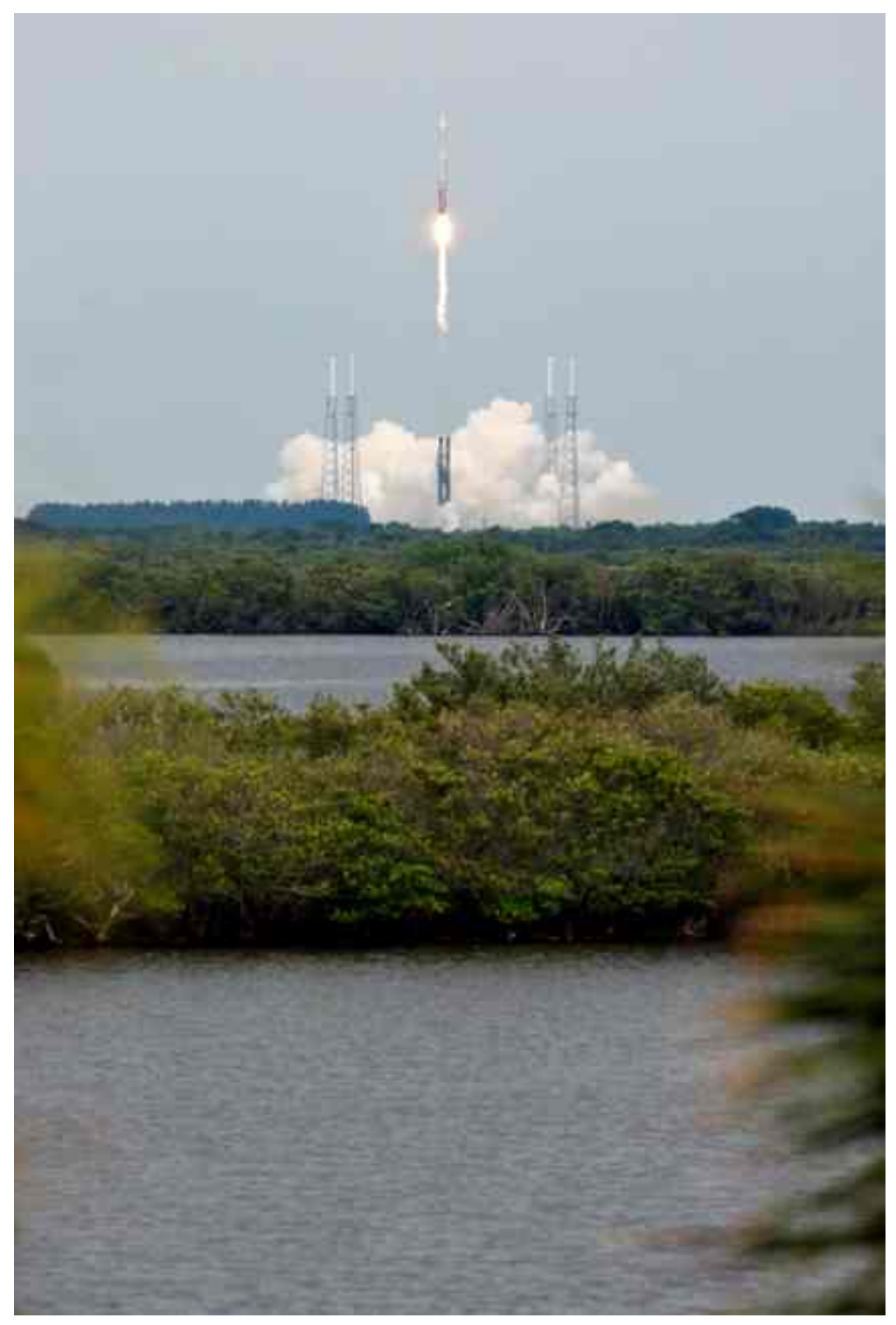

SpaceOps 2010 Conference Delivering on the Dream April 26, 2010 Huntsville, AL.

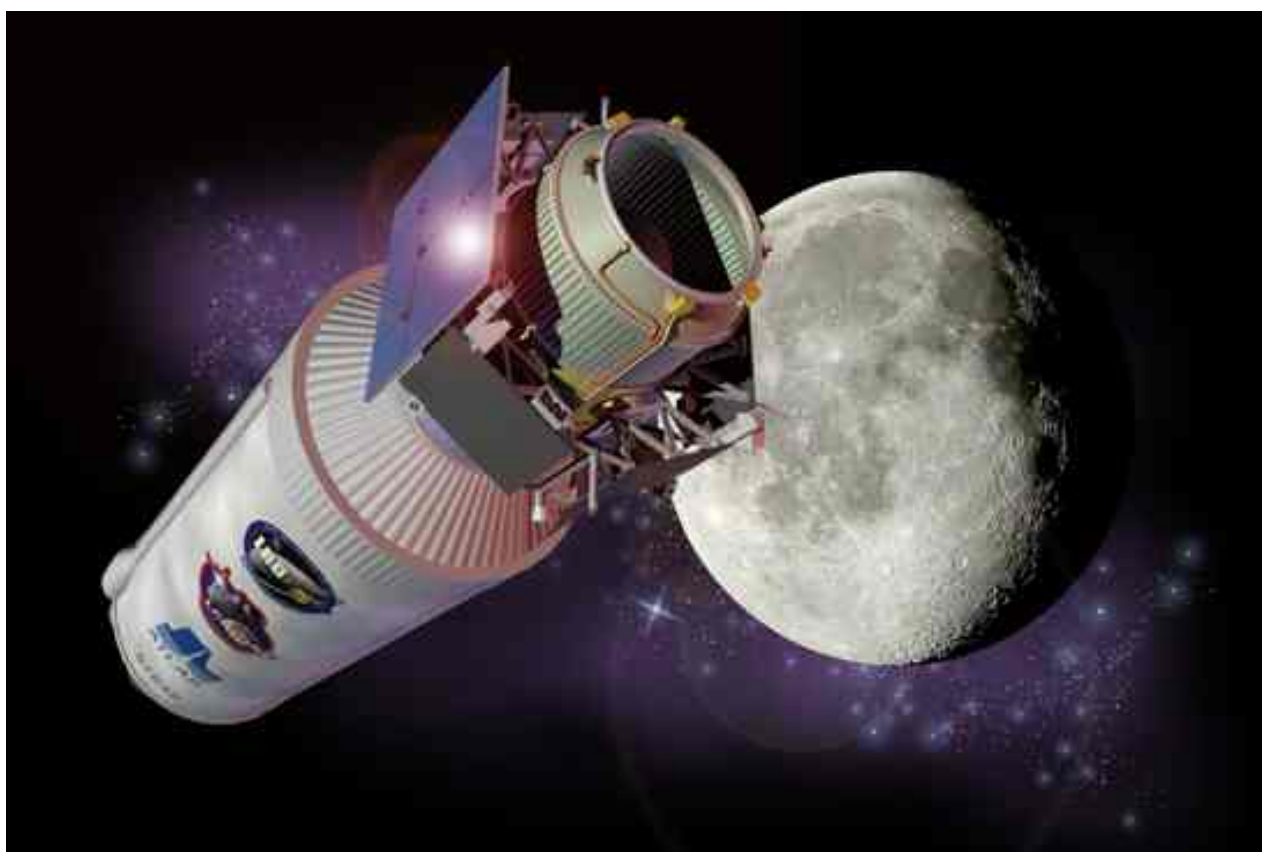

James Strong james.m.strong@nasa.gov NASA Ames Research Center 


\section{Agenda}

- Purpose of this Presentation

- Provide a few issues to watch for if and when carrying a second stage in space (Centaur in this case)

- Describe Mission

- LCROSS

- Describe applicable Mission Constraints

- Site measurements and contamination

- Describe three in-flight experiences and results.

- Centaur Venting

- Cold Bakes

- Thermal

- Summary

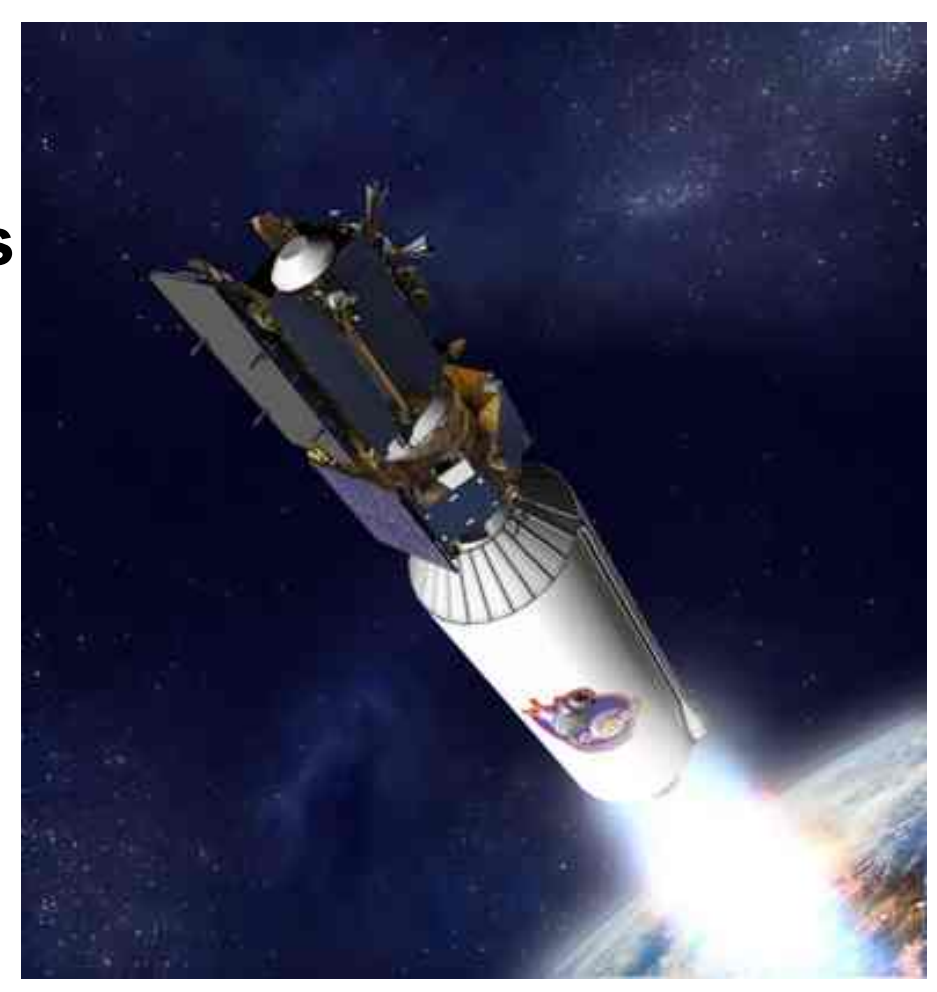




\section{Purpose of this Presentation}

- Potential reasons for carrying a second stage on an extended mission:

- Impactor

- Fabrication/hardware

- Asteroid diverter

- LCROSS Mission

- Objective to carry a Centaur second stage to moon, direct it to a target crater after $100+$ days, release it and observe the impact.

- What are some of the issues mission designers and operations teams need to deal with.

- Is the Centaur space flight ready?

- Attitude control at separation (dual ACS modes required)

- Release mechanism reliability

- Thermal control during mission.

- What should you watch out for?

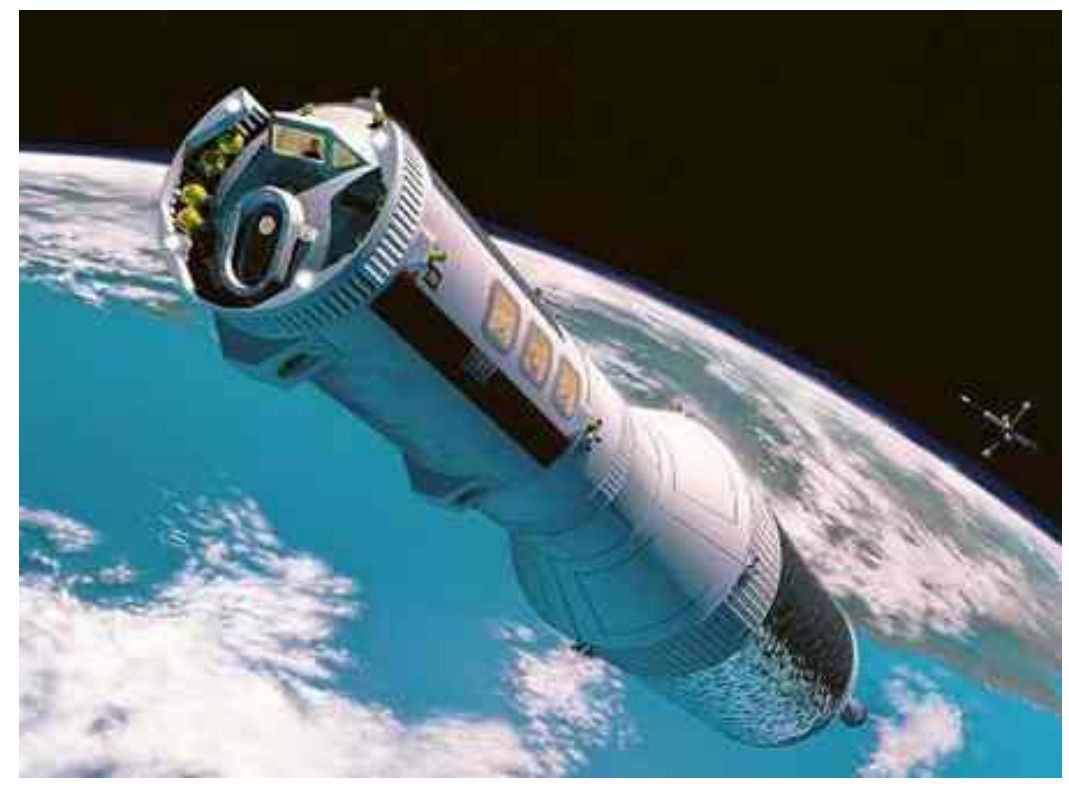




\section{LCROSS Mission}

- CLASS D (low budget, high risk tolerance), Lunar exploration

- Co-launch with Lunar Reconnaissance Orbiter on an Atlas V launch vehicle using a Centaur second stage.

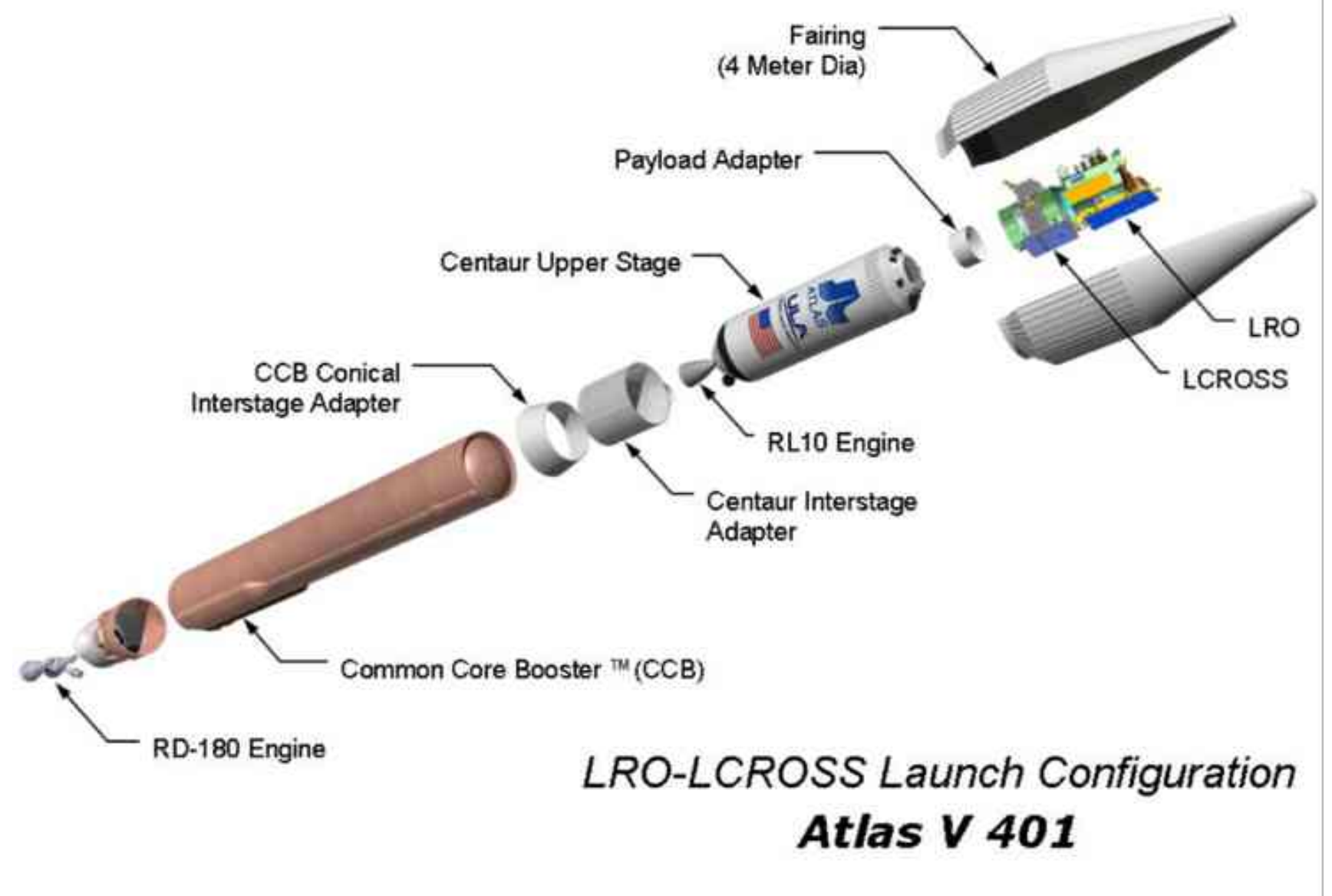




\section{LCROSS Mission}

\section{Mission Objective:}

Advance the Vision for Space Exploration by determining the possible existence of water ice at the lunar poles.

After 112 days in flight, both the Centaur upper stage and LCROSS shepherding spacecraft (S-S/C) completed the mission by impacting the moon on target in the crater Cabeus.

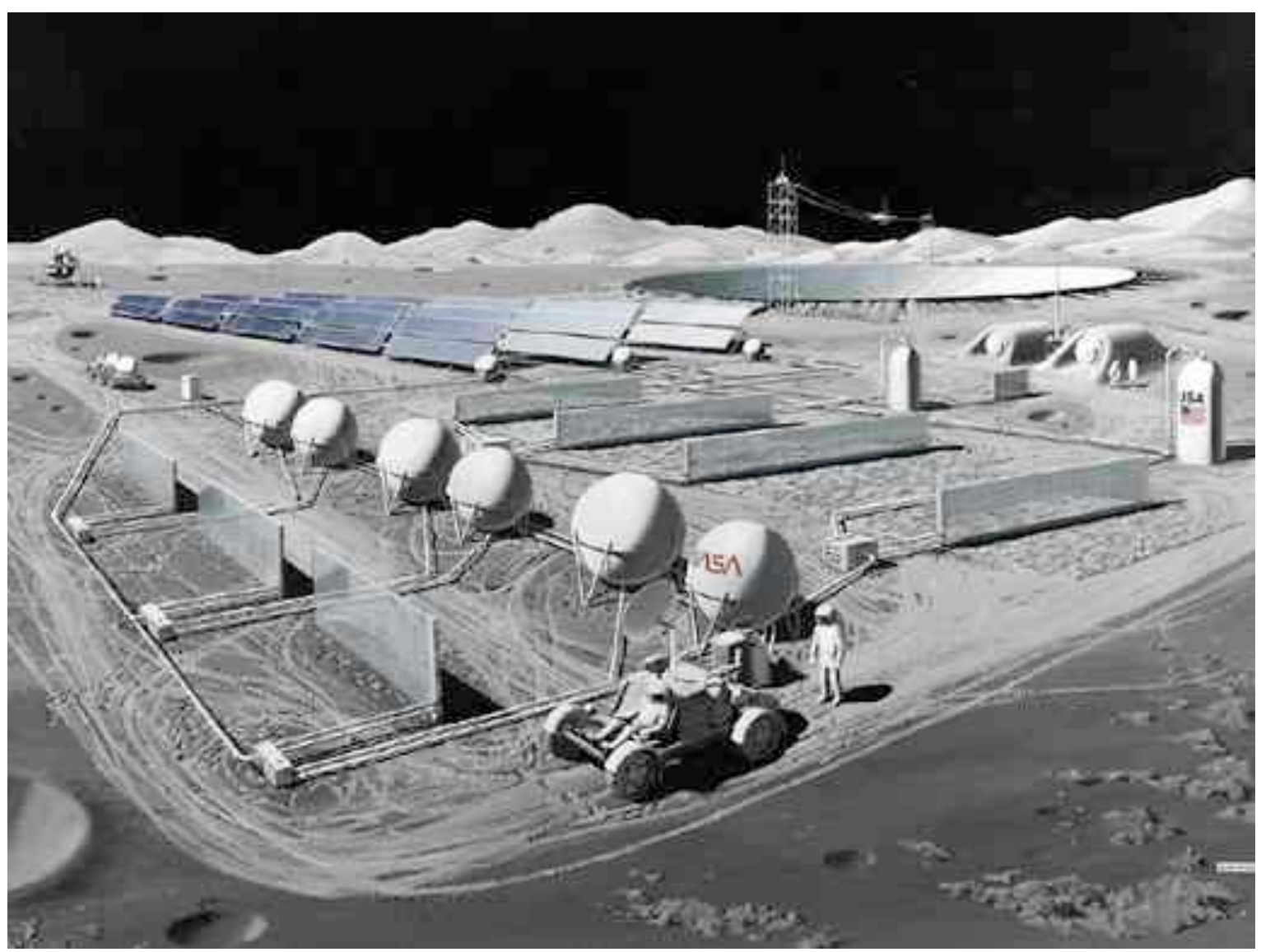




\section{LCROSS S/C}

LCROSS made innovative use of the Centaur upper stage as a primary impactor,

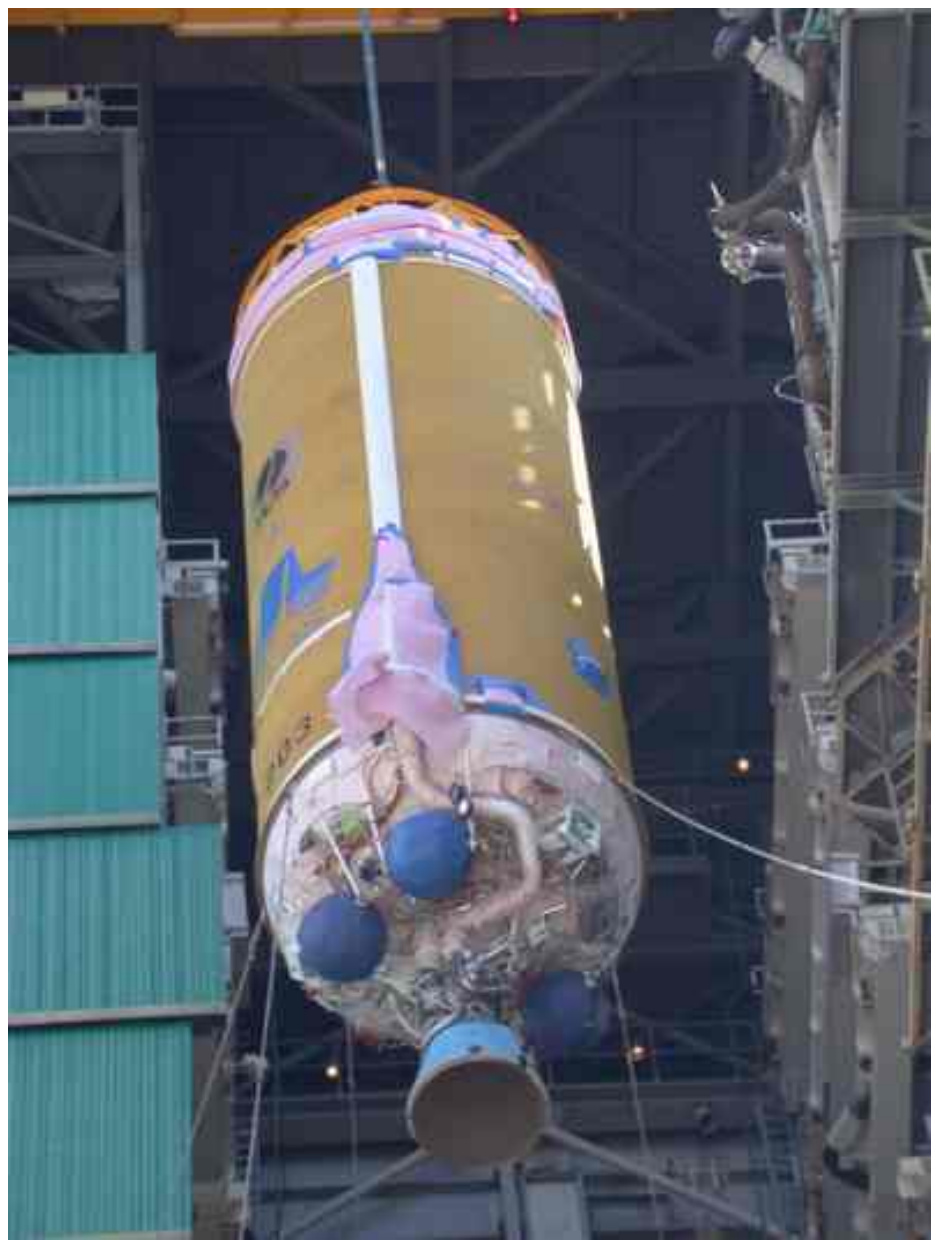

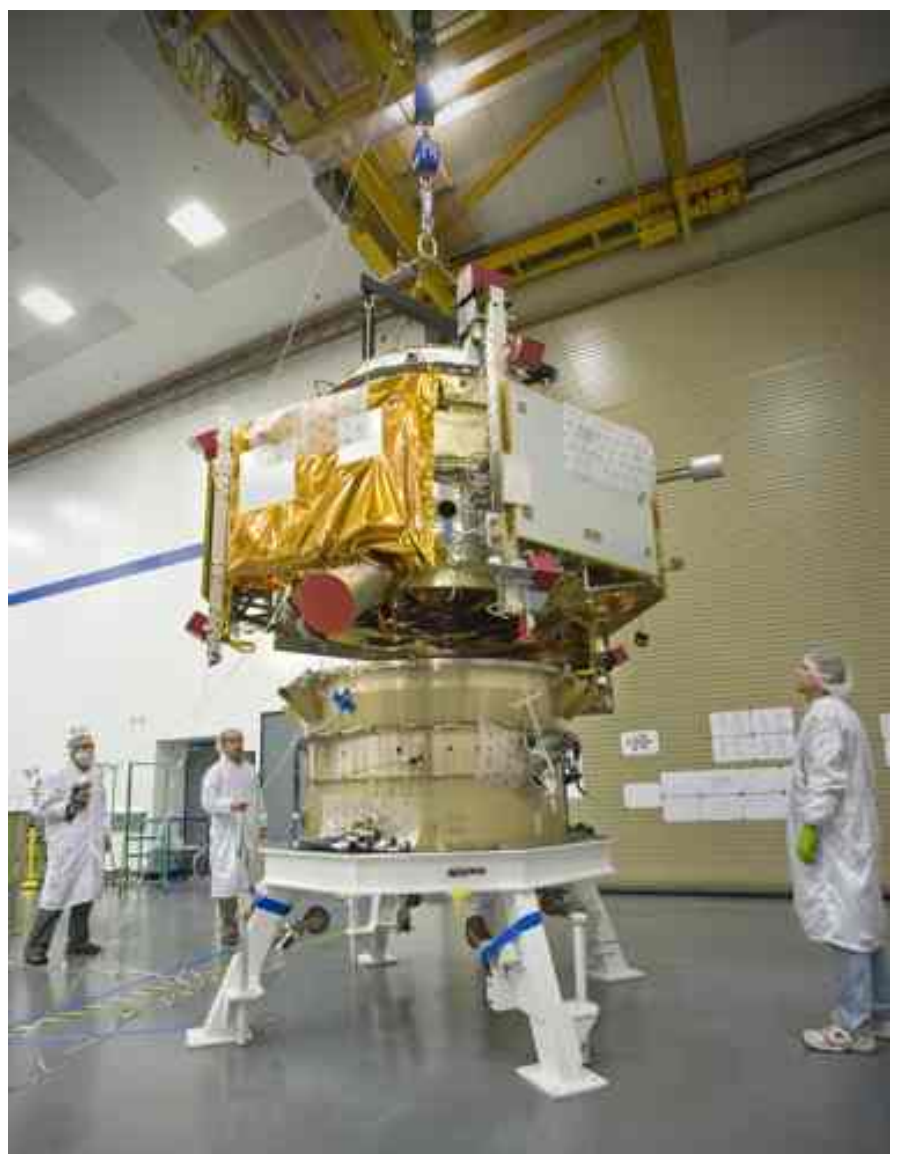

and the Evolved Expendable Launch Vehicle (EELV) Secondary Payload Adaptor (ESPA) ring as the shepherding spacecraft's primary structure. 


\section{LRO-LCROSS Launch}

LRO-LCROSS Mission Trajectory

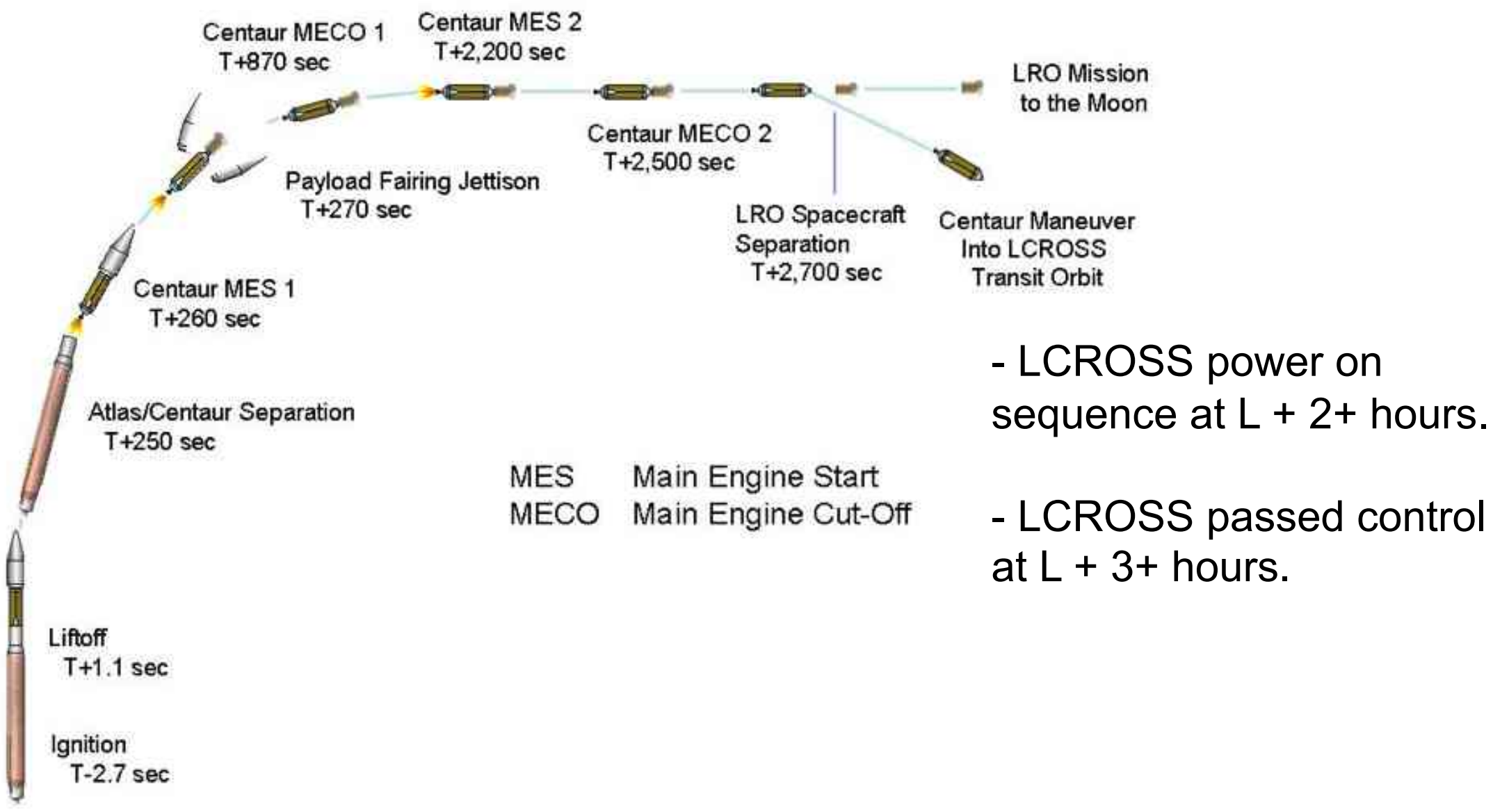




\section{LCROSS Orbit}

The LCROSS mission was focused on achieving a precise, high-energy Centaur impact within a lunar polar crater, and observing the impact and resulting ejecta plume from a combination of orbiting and Earth-based assets. To achieve the desired impact geometry, LCROSS performed a lunar gravity assist to transfer from its trans-lunar orbit to a Lunar Gravity Assist Lunar Return Orbit (LGALRO), an elliptical, Earth-centered orbit inclined steeply with respect to the lunar orbit plane.

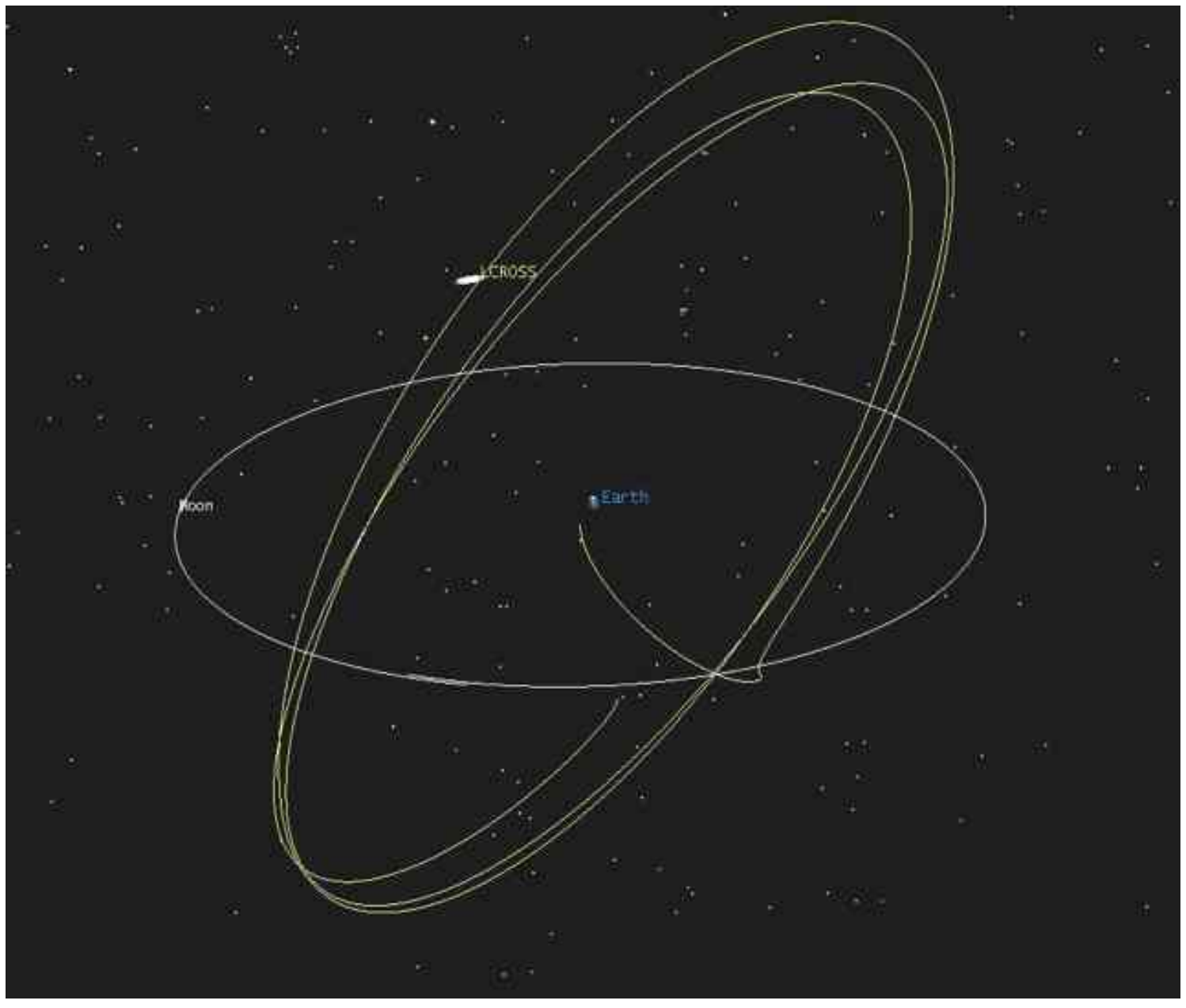




\section{Top of Orbit \#3} as of Sept 11, 2009

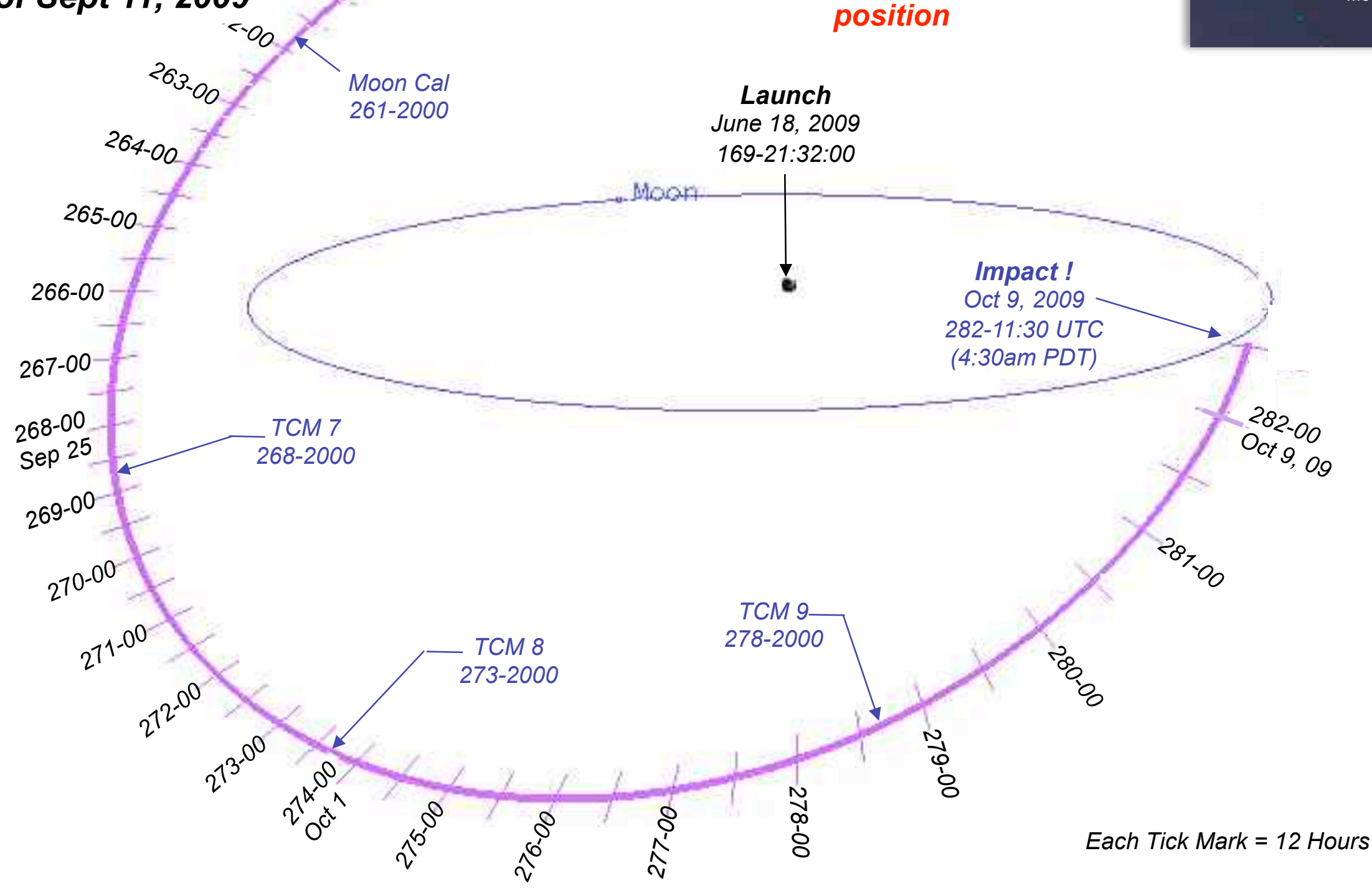




\section{Mission Constraints}

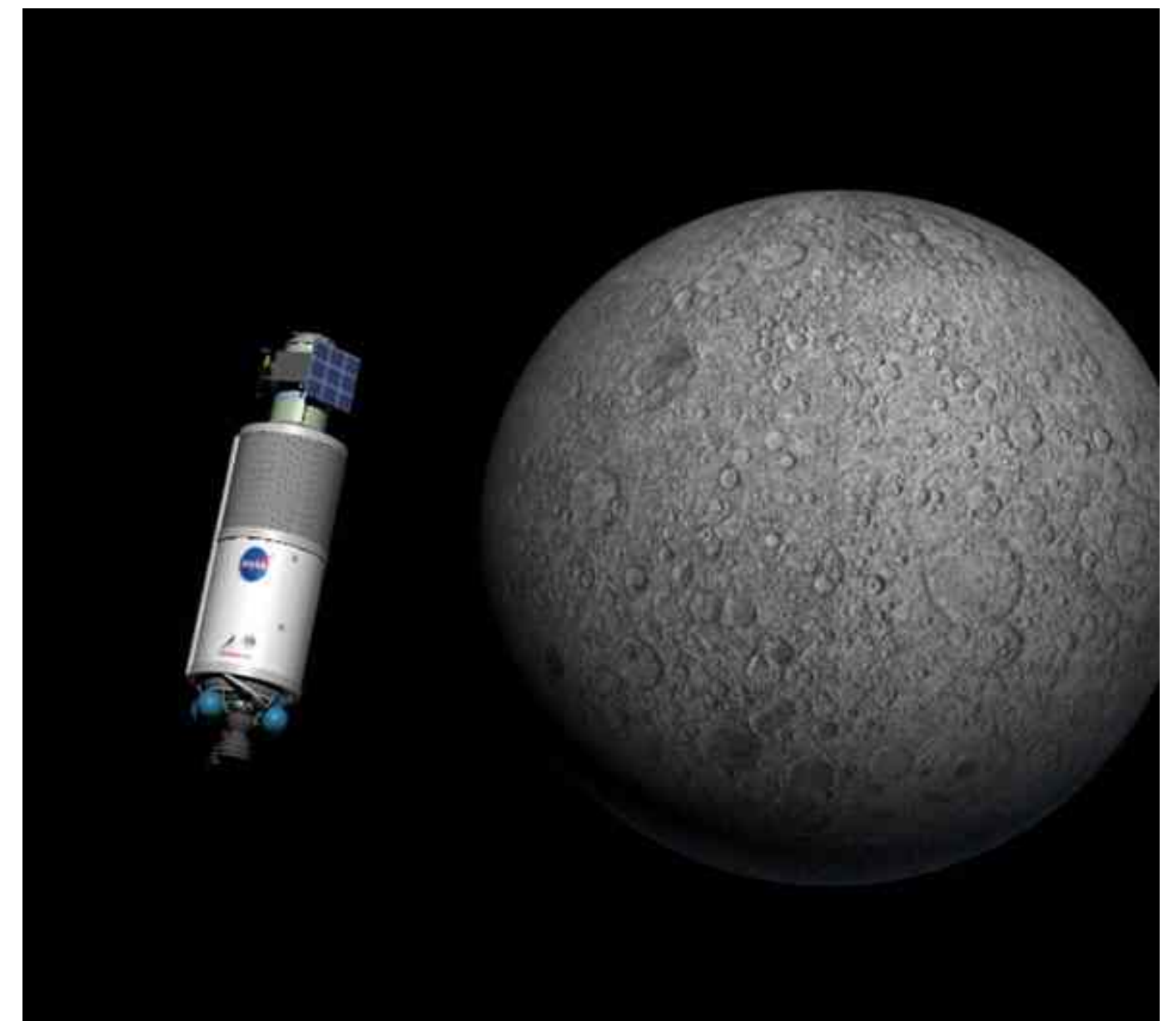

- The Launch System shall vent the $\mathrm{H}_{2}$ and $\mathrm{O}_{2}$ tanks to limit the remaining combined mass of those species to no more than $100 \mathrm{~kg}$.

- LCROSS will strive to measure the concentration of water in the ejecta cloud, as a ratio of water ice to regolith, to a level as low as $0.5 \%$ by mass, and to determine the form of which the water may be in.

- LCROSS shall distinguish water carried by the impactor from water present on the moon or provide confidence that any residual water from the impactor is scientifically insignificant for lunar water detection. 


\section{Mission \& Constraint Implications}

- Centaur systems not designed for prolonged mission operations.

- Centaur propellant systems not designed for $100 \%$ fuel and pressurant depletion, or to be contamination free.

- Propellant fill and vent valves perform well for the launch and orbit maneuver activities, but they were not designed for $100 \%$ closure after the Centaur's task is completed and products vented.
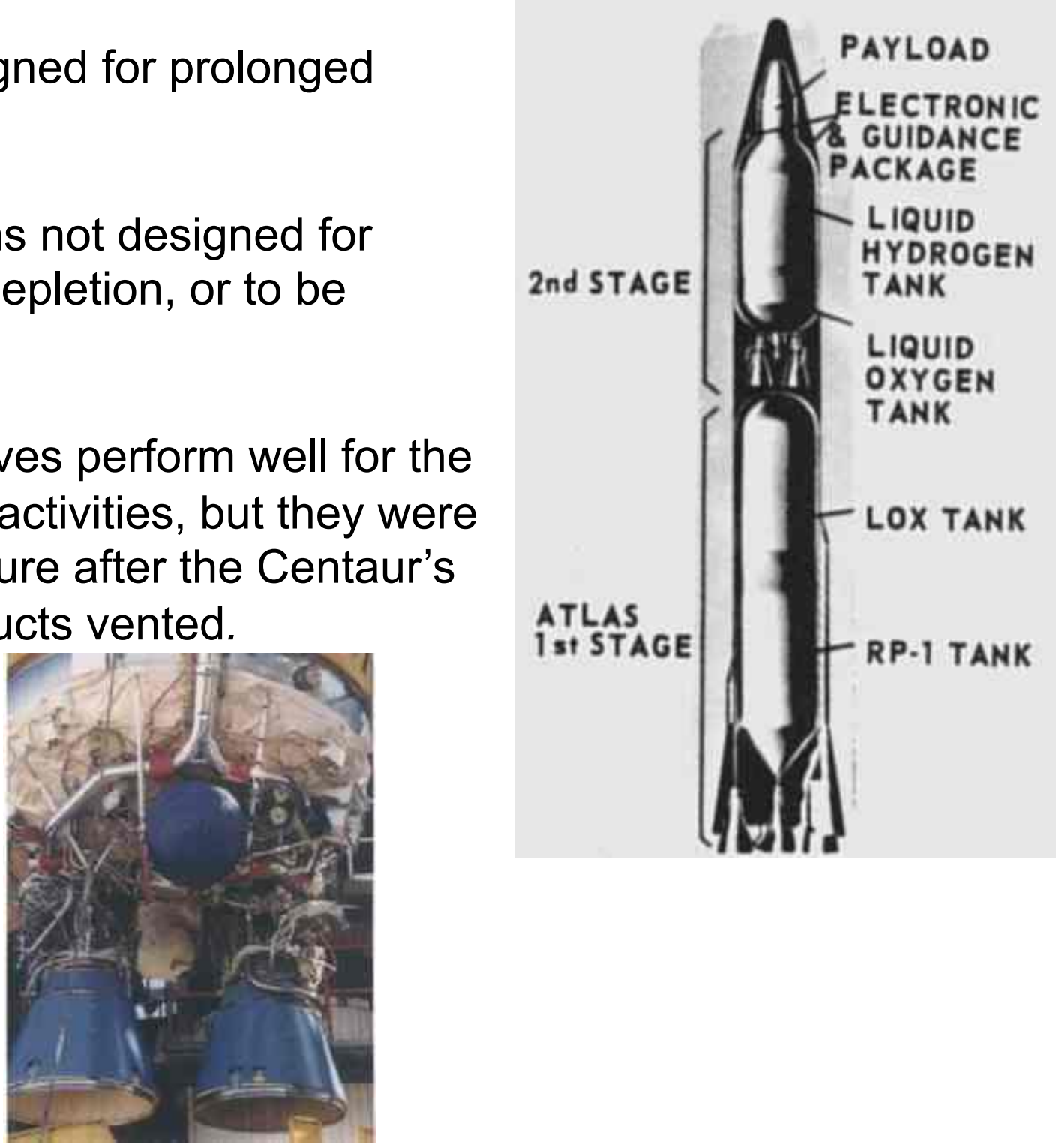


\section{Mission \& Constraint Implications}

- Centaur materials and insulating foam not designed to be contaminant or water free. No normal operation designed for removal.

- Centaur payload release mechanism not designed to function after many days in space. Reliability of this function an unknown.

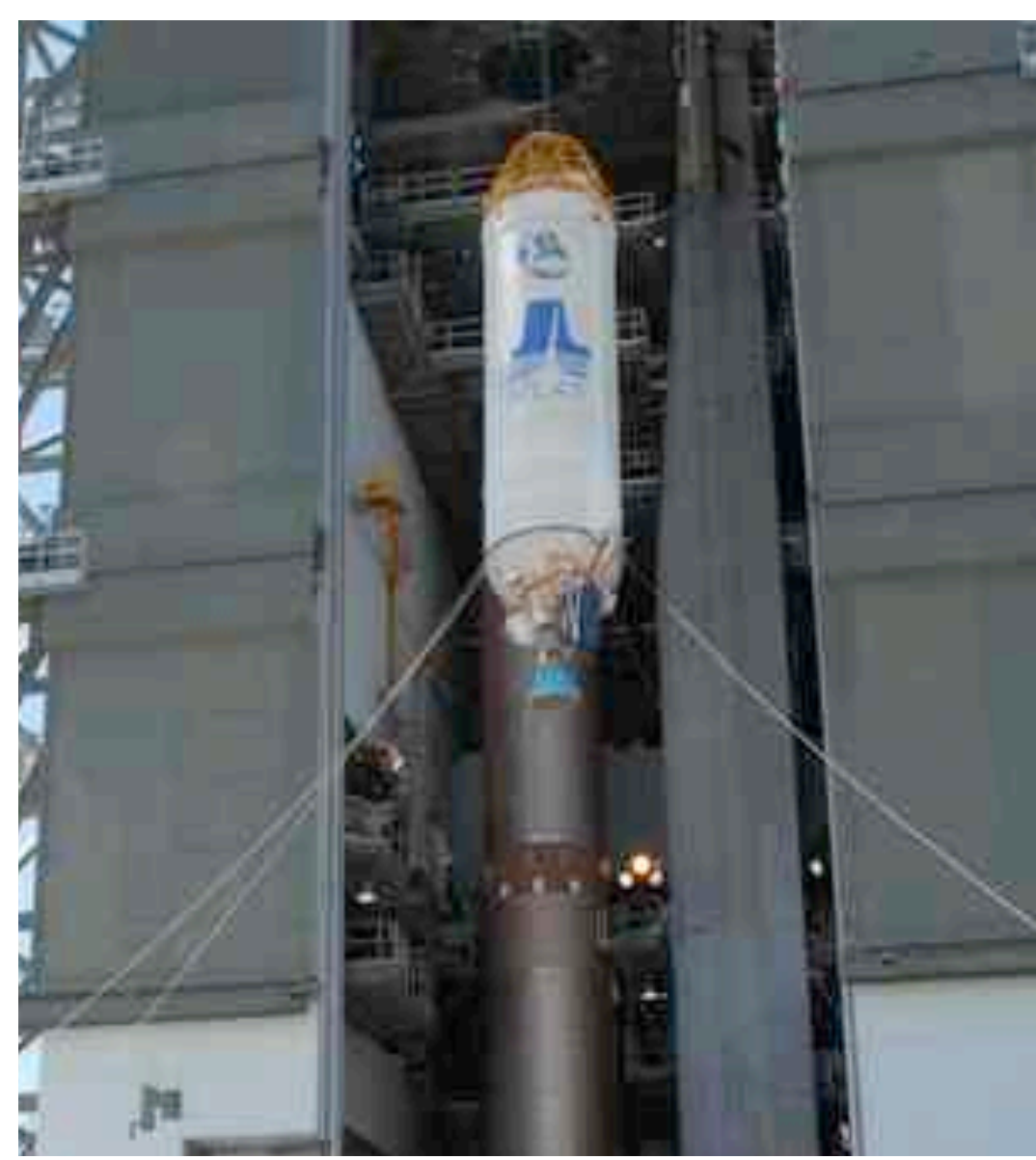




\section{Item \#1: Centaur Leak - Problem}

- Project assumed a potential for residual propellants to leak from one or more valves on the spent Centaur stage for weeks or even months following launch.

- Any residual fuel still remaining could vent and induce torques on the S-S/C and Centaur system. This generated a significant risk for LCROSS.

- Reliably predicting the amount of S-S/C propellant needed to counteract venting disturbance was difficult because of the following: uncertainty in the magnitude of the leak rate; direction that the escaping gasses would take (depending on number of valves open, their location, and effective thrust parameters including leak vector); and the decay time associated with such a leak.

- Under low and moderate leak rates, analysis showed that the nominal control mode designed for the cruise portion of the mission (lasting on the order of 100 days) would consume all the propellant $(305.5 \mathrm{~kg}$ launch load) before completing the mission. 


\section{Item \#1: Centaur Leak - Mitigation}

- Required Operations team to develop a number of operational strategies for assessing leak severity and alternate modes of operating the vehicle to counteract any leak effects.

- Adapting the cruise control gains to work with increased thruster pulse width (50ms to $100 \mathrm{~ms}$ ), increasing the low duty cycle efficiency of the pulses.

- Changing the thruster selection logic to fire thruster pairs instead of quads (fuel savings at expense of orbit determination accuracy).

- A new ACS mode entailed spinning the vehicle about its axis of maximum inertia with the solar array (vehicle $-Y$ body axis) pointed at the sun.

- The following two plots show the results from torque observations to assess leak effect collected 19.5 and 26.5 hours after launch. These were made with thruster control inactive and show a good drop off in leak torque. 


\section{Item \#1: Centaur Leak - Results}
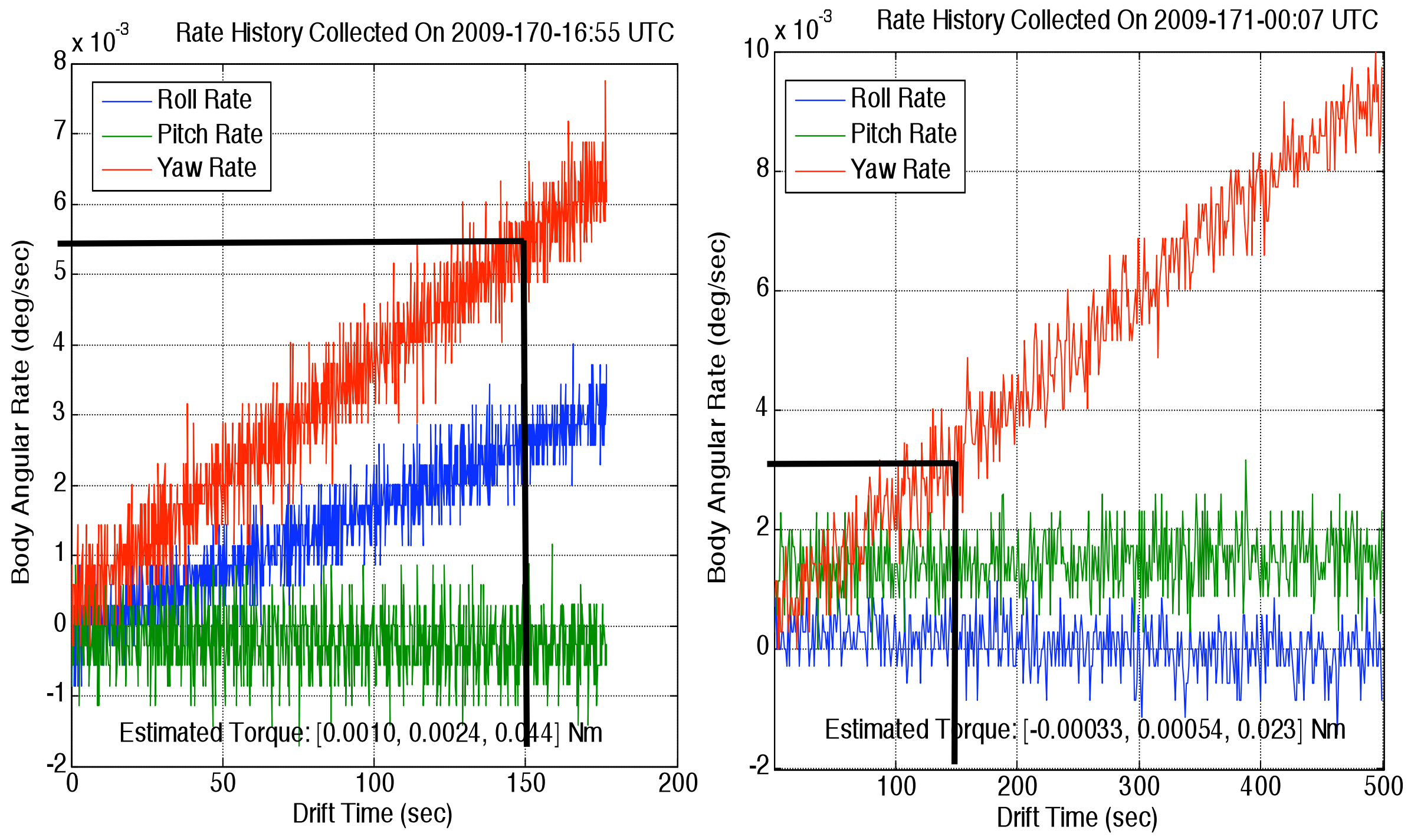


\section{Item \#1: Centaur Leak - Lessons}

- These efforts expended significant resources from many groups at a time when launch preparations and operations tests were in progress.

- Risk posed by potential Centaur leak rates influenced the decision to slip the LRO/LCROSS launch by two weeks.

- Conclusion:

When flying systems not designed for extended use :

- Expect to spend extra effort in the discovery, analysis and planning for newly discovered behaviors.

- Investigate all potential behaviors of the system to be launched and flown as early as possible to anticipate problem behavior and to develop and test strategies to counteract them. 


\section{Item \#1: Centaur Leak - Burps}
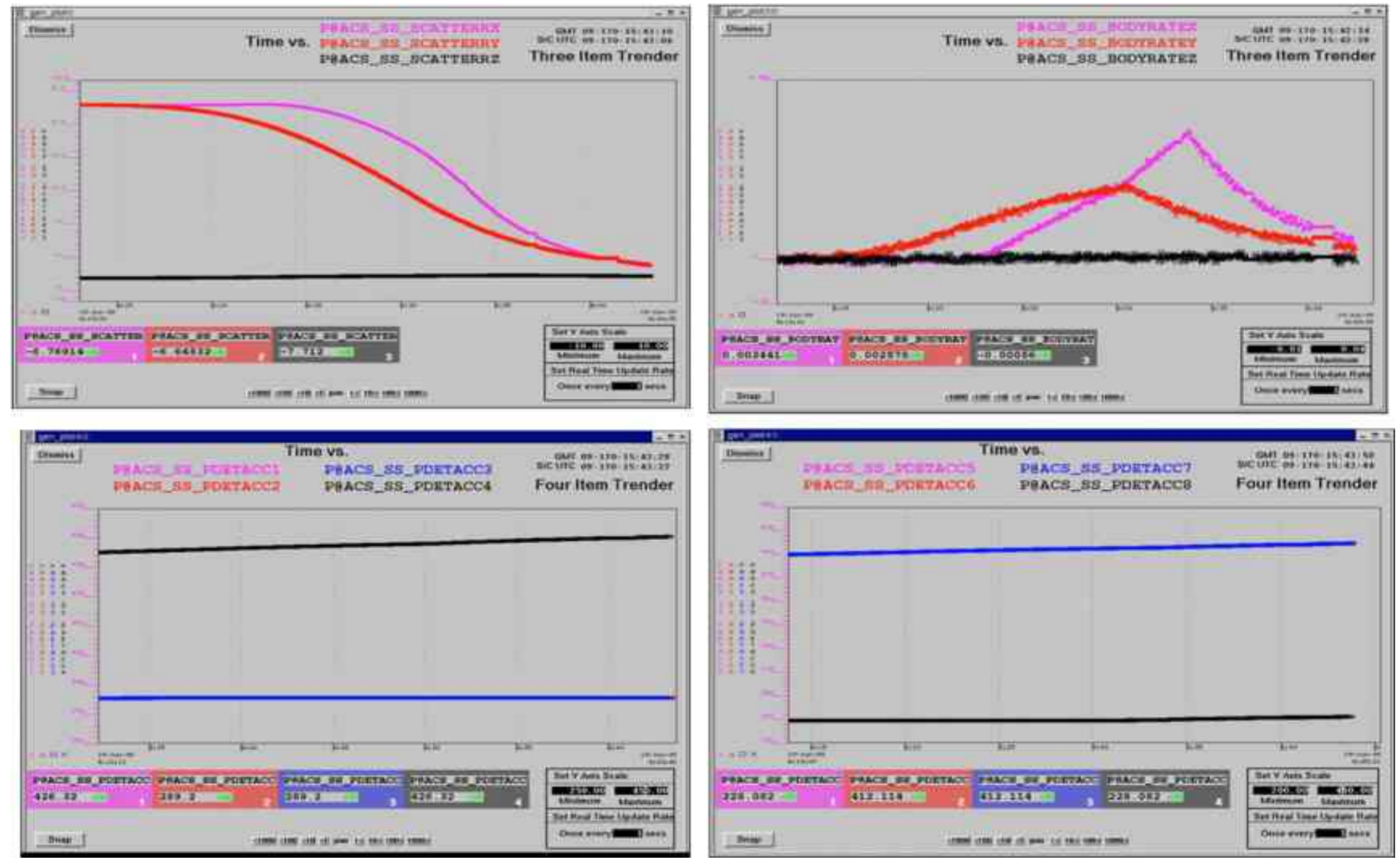

3-axis body rate and attitude error (upper plots) and 8 thruster outputs (below) versus time showing unexpected torque changes during cruise. 


\section{Item \#2: Centaur Water - Problem}

- Water embedded in the Centaur insulating foam or attached to the vehicle surface was a known threat for both science contamination of the impact site as well as final impact targeting.

- As water was the primary science objective, any remaining in or on the lunar impactor could significantly interfere with or invalidate science conclusions.

- After S-S/C separation, any significant amount of water remaining on the Centaur (even if within science contamination requirements) had the capability to alter the Centaur course prior to impact. As the Centaur tumbled or rolled prior to impact with no S-S/C control, it would expose its 'cold side' to the sun, and subsequent water outgassing would impart vectored thrust, throwing it off target.

- Per ULA outgassing analysis, there could be $\sim 1 \mathrm{~kg}$ of water trapped in foam, half of which could be boiled off when coldside was exposed to the Sun.

- Per same ULA analysis, $90 \%$ of this water would bake out within one hour of exposure. 


\section{Item \#2: Centaur Water - Mitigation}

- Analyze and quantify sources of water in and on the Centaur stage prior to launch.

- Design operational flight procedure to 'Bake-Out' water ice from the Centaur insulating foam while in cruise.

- Expose the cold, anti-sun side of the Centaur temporarily to the sun with the objective of removing water on the surface and in the foam.

- Verify no further outgassing was present, or, estimate the quantity of water remaining based on delta- $V$ and pre-flight estimates.

- Use precise Doppler measurements taken during CSB maneuvers to assess effectiveness of the maneuvers.

- Three Cold-Bakes performed in 73 days

- Mixed with other mission tasks and science calibrations.

- Each execution was unique in design and result.

- Doppler results from first bake on next chart.

- Showed good evidence of outgassing, and usable results. 


\section{Item \#2: Cold Side Bake Doppler signature (CSB\#1)}

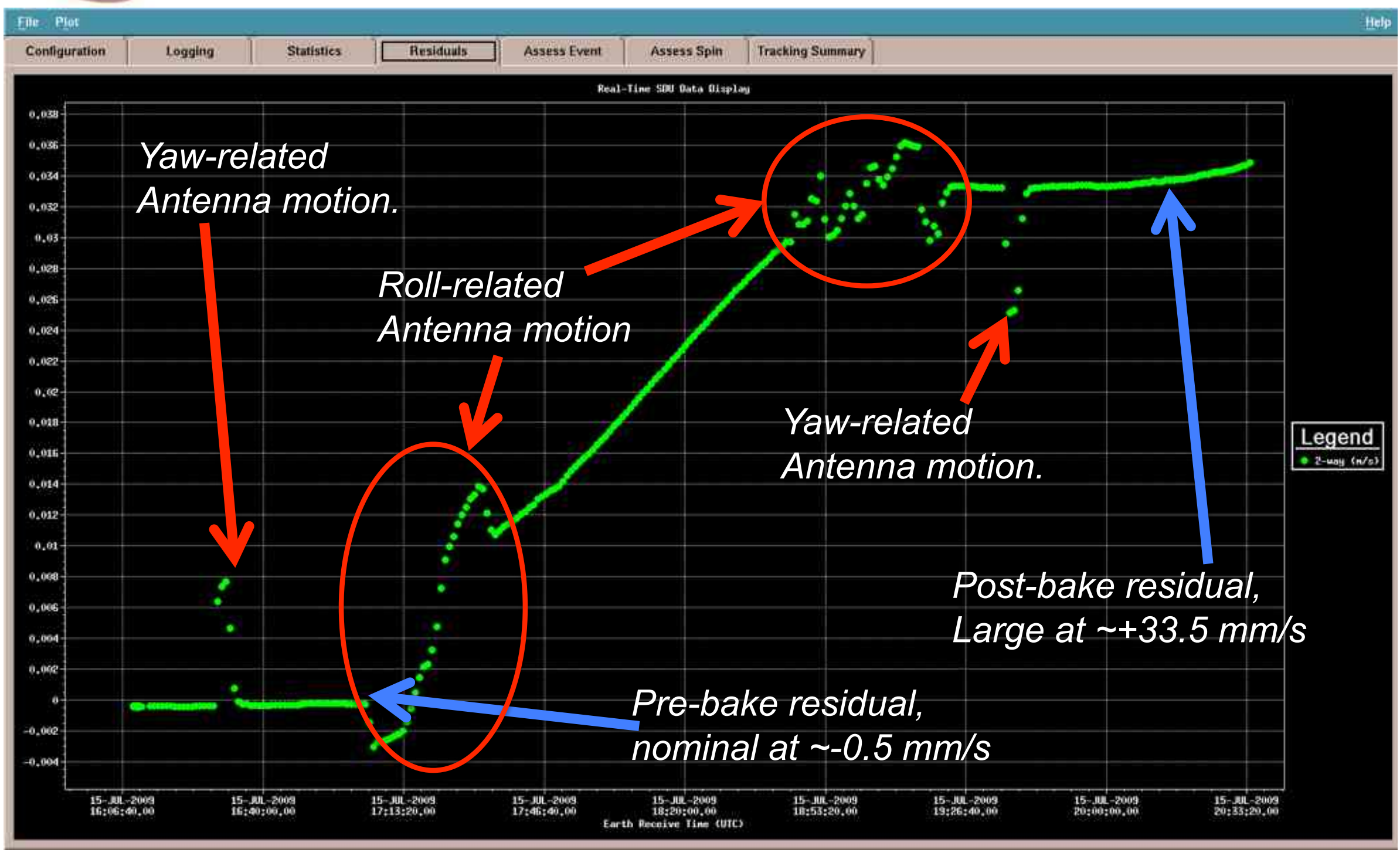




\section{Item \#2: Cold Side Bake Results}

- For CSB \#1, spacecraft velocity shift was $\sim 3.4 \mathrm{~cm} / \mathrm{s}$ correlating directly with the bakeout period, which was three times higher than expected.

- There was no appreciable change in the rate of change in velocity, implying that offgassing material present was extensive and might take many hours to deplete.

- The transients in the acceleration seemed to be very brief, ie; it did not take much time for the dark-side to "warm-up" before seeing an effect on the trajectory, and it did not take much to time for the acceleration to diminish once it returned to shadow.

- Outgassing resulted in all Three axes driving to and 'sticking' to attitude deadbands.

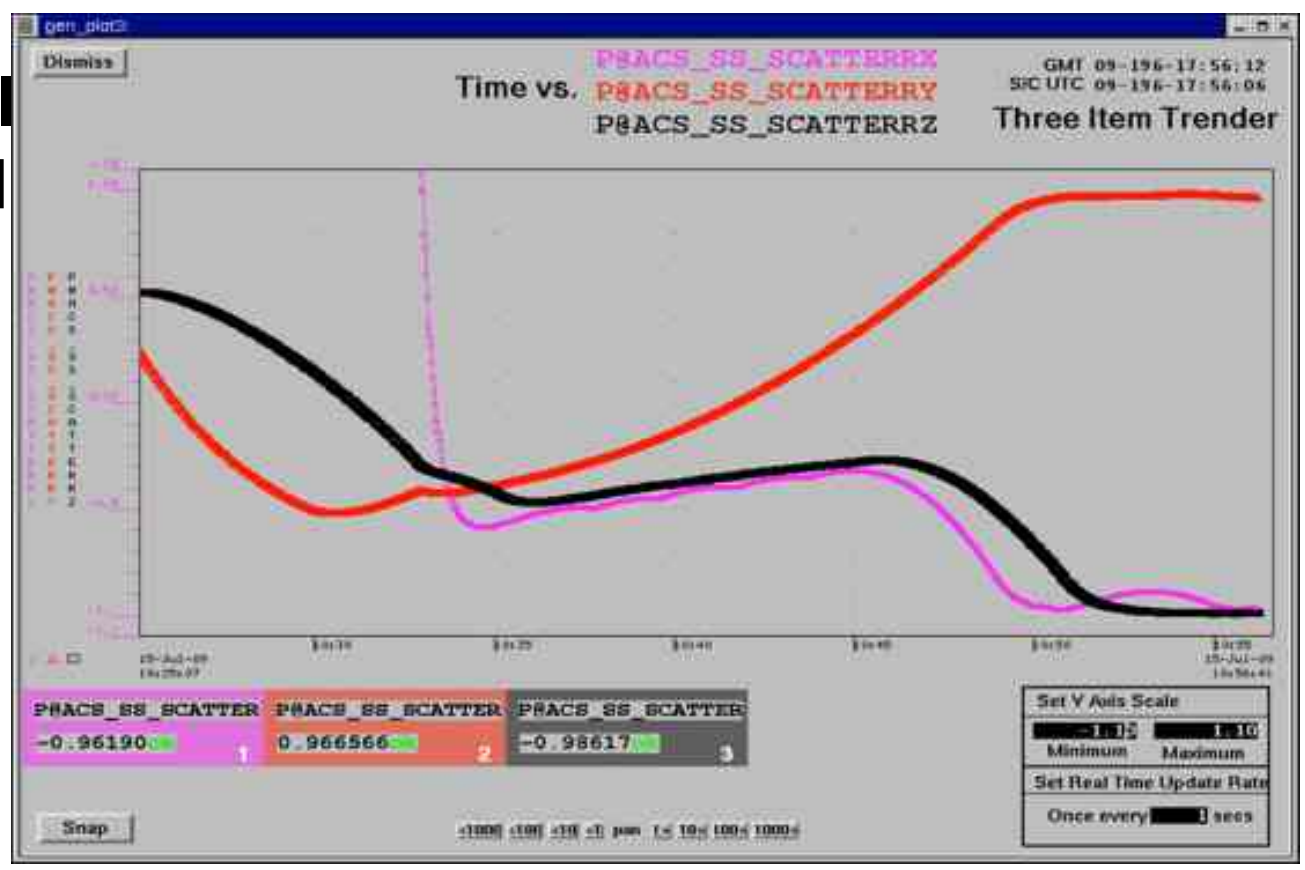




\section{Item \#2: Cold Side Bake Signature (CSB\#2)}

\section{Doppler signature (CSB\#2)}

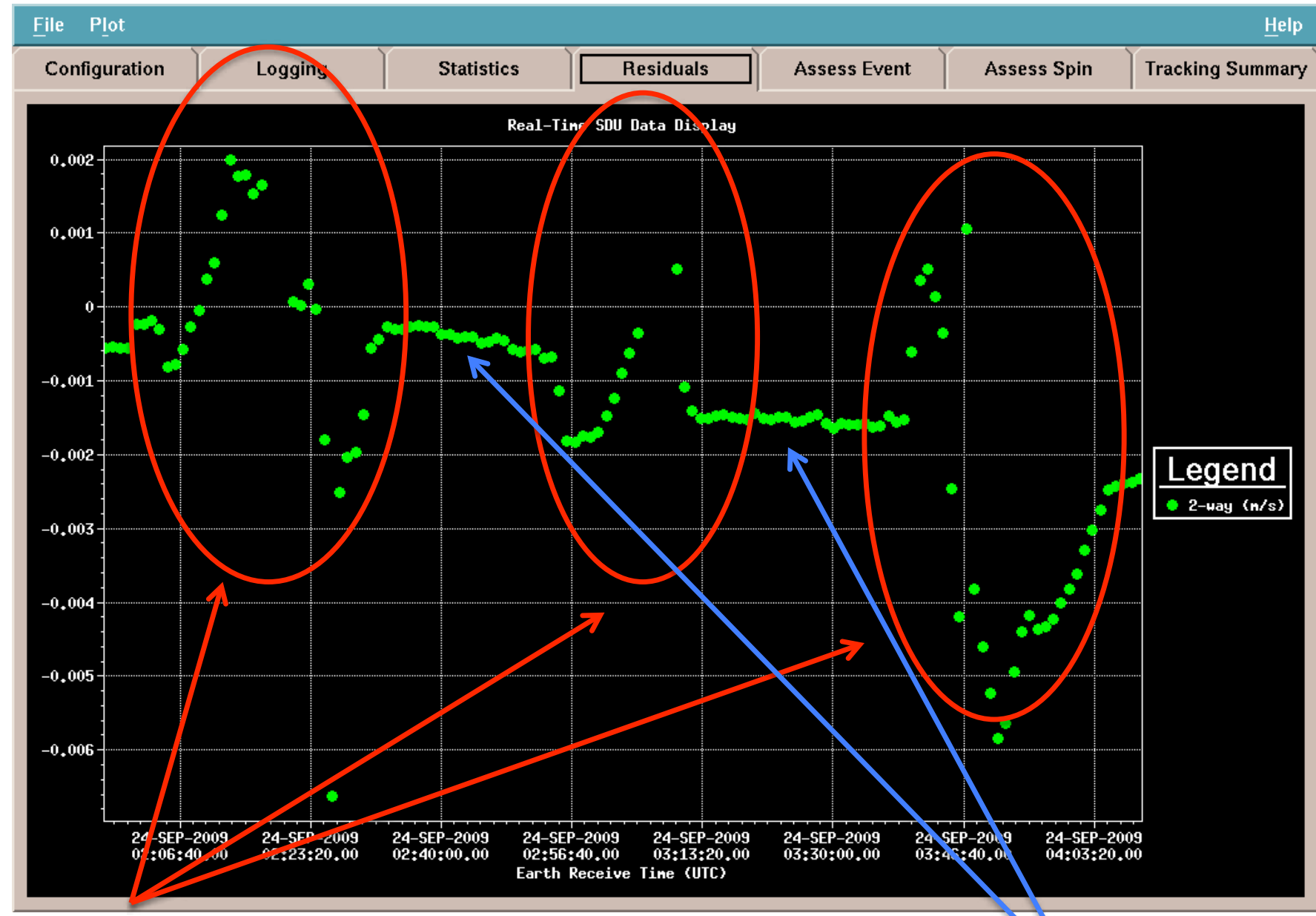




\section{Item \#2: Centaur Water - Summary}

- Bakeouts determined (to the extent measurable) that Cold Bake maneuvers had removed Centaur water.

- No residual cooking off of ice seemed to have occurred during cruise between CSB\#2 and CSB\#3 as these two showed similar trends.

- $\quad 20 \%$ of the expected delta-v was observed on CSB\#2, which implied a cliff in the outgassing effects was passed and/or additional cookoff of cold-side material occurred for hours/days after CSB\#1.

- Post separation targeting errors, initially large, became acceptable after three bakeouts.

- The Centaur impactor target error after CSB\#1 was at the 1-2 kilometer level as determined from Doppler residual data. ( $1.75 \mathrm{Km}$ required)

- Target error after CSB\#2 \& \#3 was bounded at 410 meters.

- Pointing error at impact due to OD was expected to be $0.25^{\circ}$

- When added with other OD error, there was no margin for OD pointing error performance.

- This was mitigated by nominal braking burn performance and use of additional ground station tracking data. 


\section{Item \#2: Centaur Water - Lessons}

- Results from these Cold Bake maneuvers validated pre-launch concerns of water contained on the Centaur vehicle and resulted in successful depletion of remaining moisture to acceptable levels.

- Conclusions:

When flying systems that may require special operations (as in anti-contamination maneuvers here):

- These maneuvers required significant effort from and exercised all components of the mission operations team.

- Pre planning for these events was therefore crucial in the mission operations planning and testing phases.

- Look for these potential extra mission requirements during operations design. 


\section{Item \#3: Thermal - Problem}

- Pre-launch thermal analysis (margin accuracy) and in-flight thermal control due to:

- Thermal sink of the large Centaur mass.

- Radiative and reflective properties of the Centaur.

- Large potential sun shadow created by the Centaur.

- Coatings or surface preparations that could potentially flake or have thermal property changes over mission duration.

- Other concerns requiring analysis (if possible).

- Thermal characterization of the Centaur's mating electrical connector capability.

- Life and function of the separation clampband after prolonged cold.

- Potential leak or burst hazard of Centaur batteries while in proximity to the LCROSS S/C solar array.

- Mitigation:

- Analyze where and as much as possible. Develop dynamic model.

- Determine behavior in flight and adapt as needed. 


\section{Item \#3: Thermal - Results}

Typical thruster valve temps in cruise.

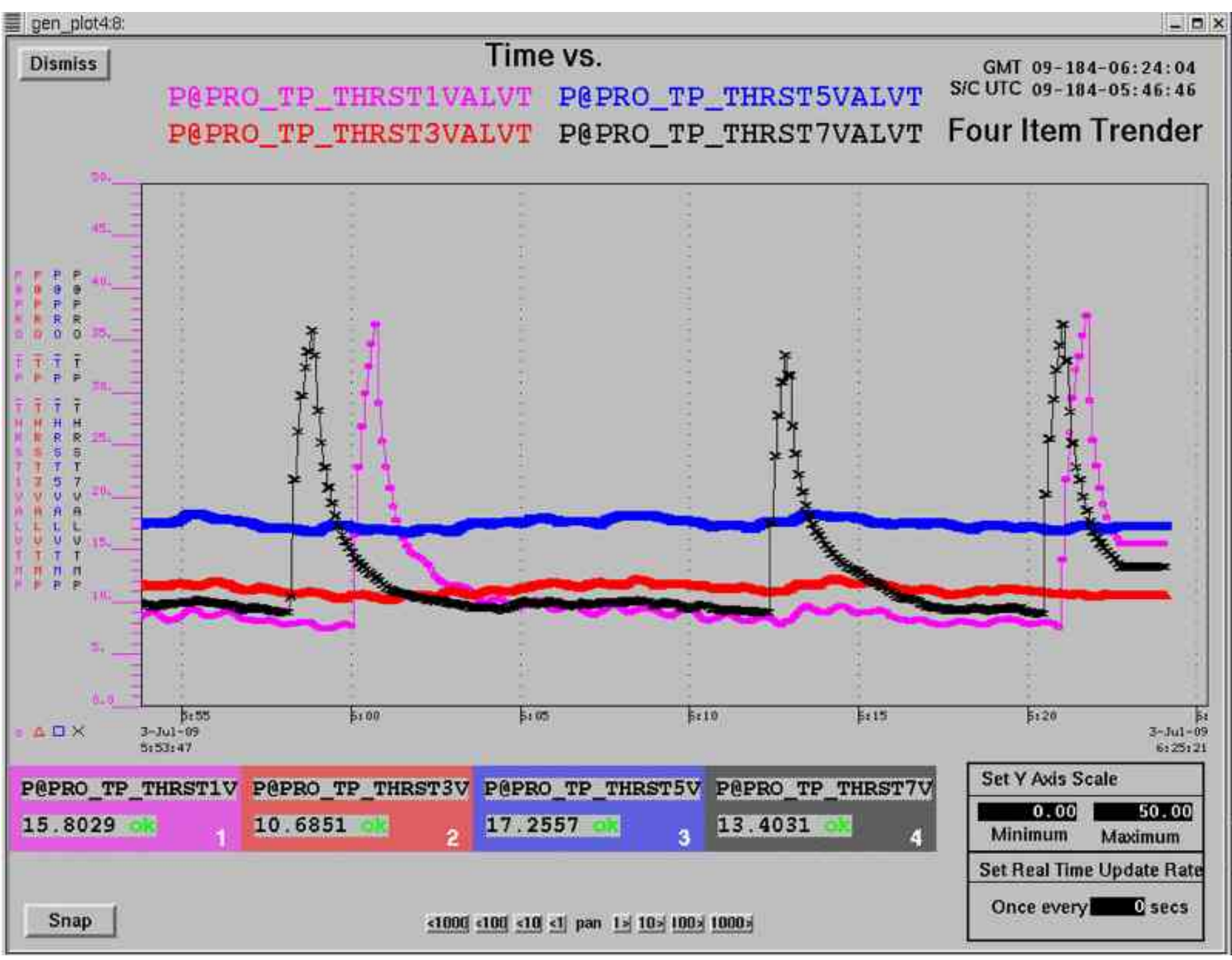




\section{Item \#3: Thermal - Example}

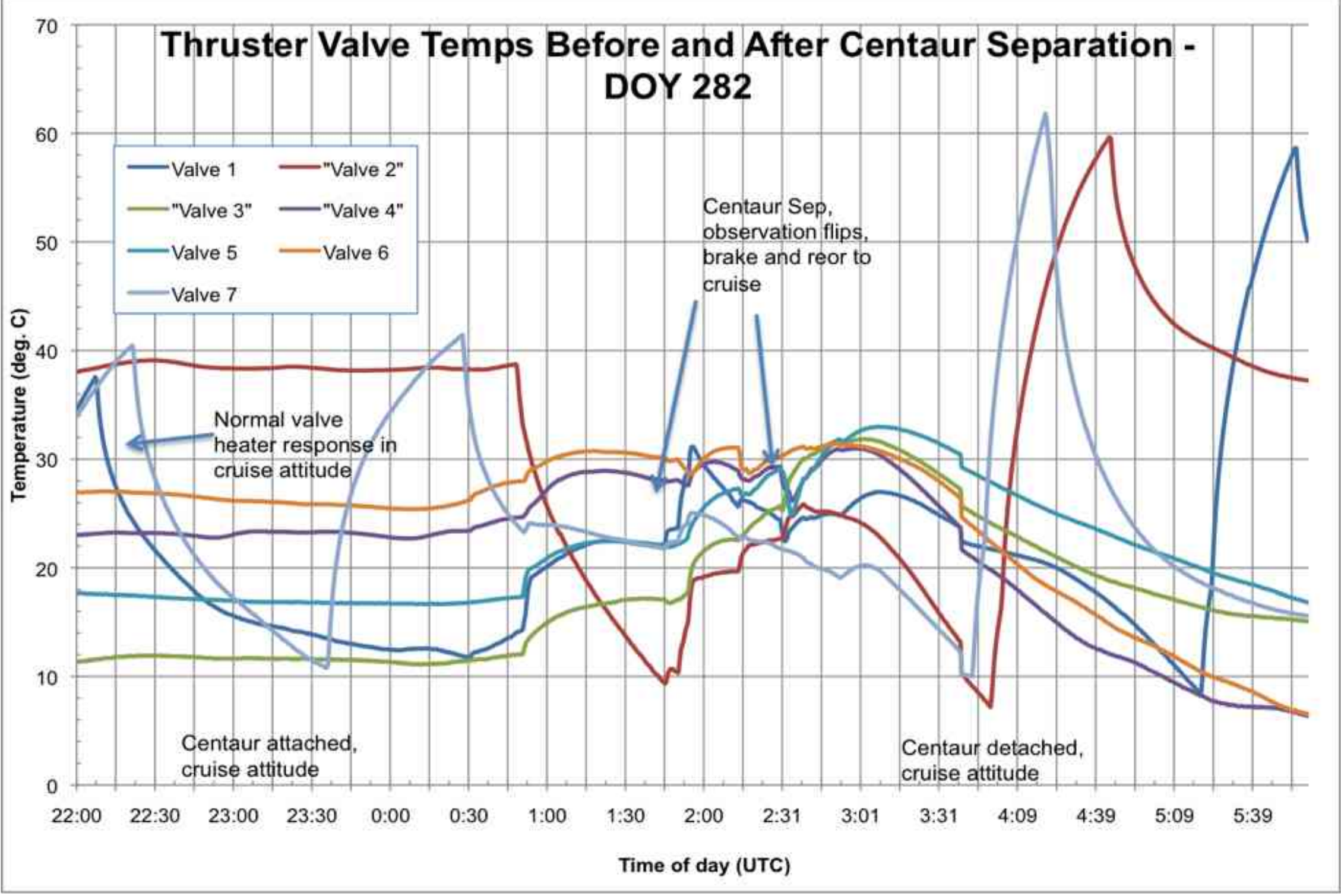




\section{Item \#3: Thermal - Lessons}

- A good thermal analysis tool and models is required for proper performance and minimal surprises. Given class $D$ budget constraints, very important to choose where to spend analysis effort.

- Conclusions:

When flying systems that may have unique and complicated thermal properties:

- Determine where to spend thermal analysis efforts to best mitigate your largest expected risks.

- Do plan for flight operational procedures that can compensate for wide variations in thermal response.

- Exercise diligence and good risk analysis when determining the level of uncertainty of these effects. 


\section{Summary}

- Future projects may find many different uses for spent upper stages as part of their missions.

- The LCROSS project was impacted significantly by Centaur shepherding considerations.

- The unknowns:

- How do you make a second stage vehicle and its systems fully compliant with your mission requirements \& constraints?

- Where do you spend your analysis and operations resources?

- Back to good basic practices:

- Work with (launch) system provider early to determine and analyze risks.

- Do exercise good analysis for determining uncertainty and risk.

- Look for unique mission requirements resulting from new flight hardware.

- Search for and anticipate unique problem behaviors early in design to develop and test strategies and flexible flight procedures to counteract them.

- Anticipate extra resource allocation needed for design and operation of new, unique or unproven space flight systems.

- The upper stage system (Centaur in this case) must be part of the project design and operations process development. 


\section{'Cause you never know......}

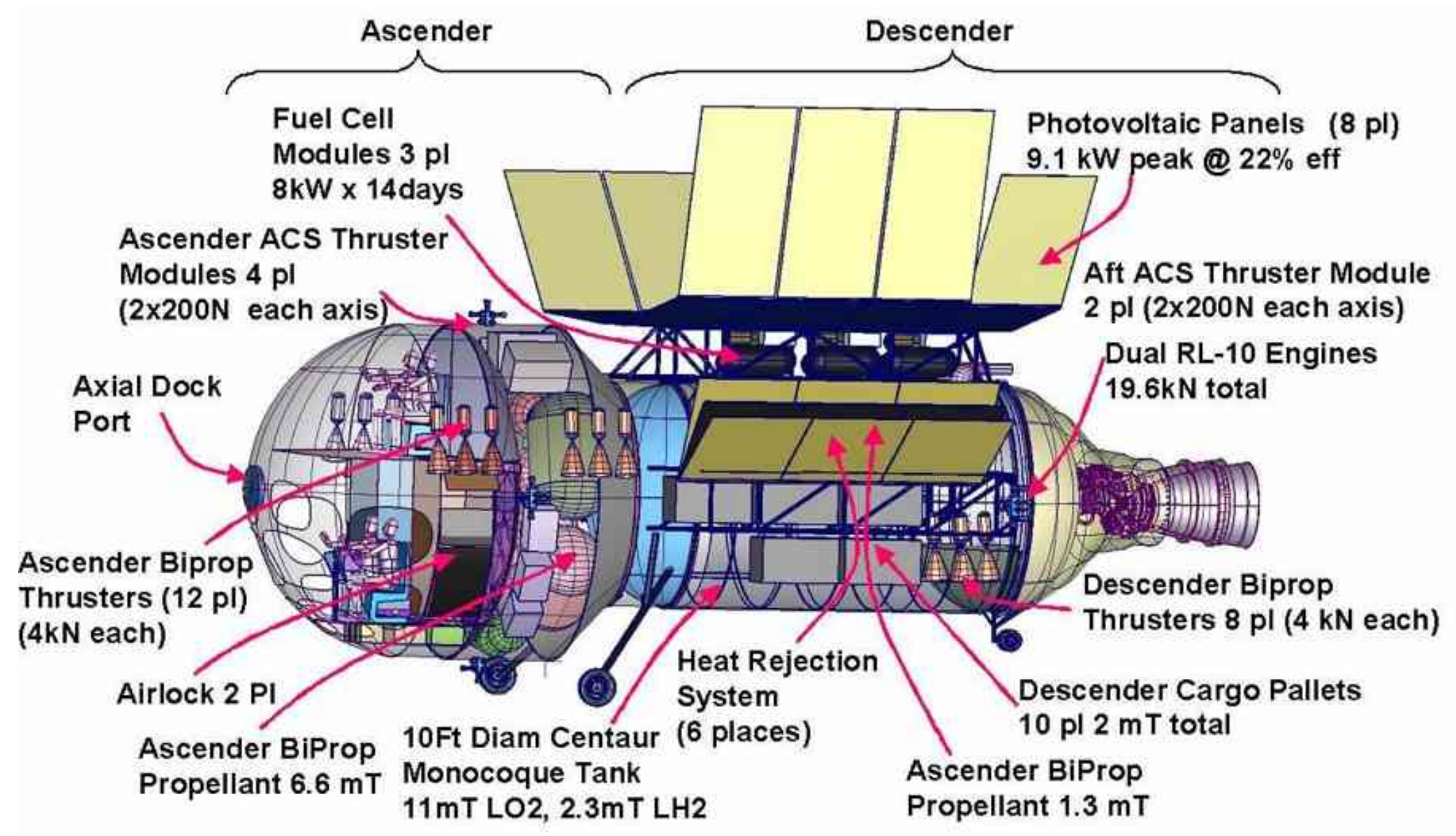

Centaur Application to Robotic and Crewed Lunar Lander Evolution

AIP Conf. Proc. -- January 30, 2007 -- Volume 880, pp. 779-786 SPACE TECHNOLOGY AND APPLICATIONS INTERNATIONAL FORUM-STAIF 2007: 11th Conf Thermophys.Applic.in Micrograv.; 24th Symp Space Nucl.Pwr.Propulsion; 5th Conf Hum/Robotic Techn \&Vision Space Explor.; 5th Symp Space Coloniz.; 


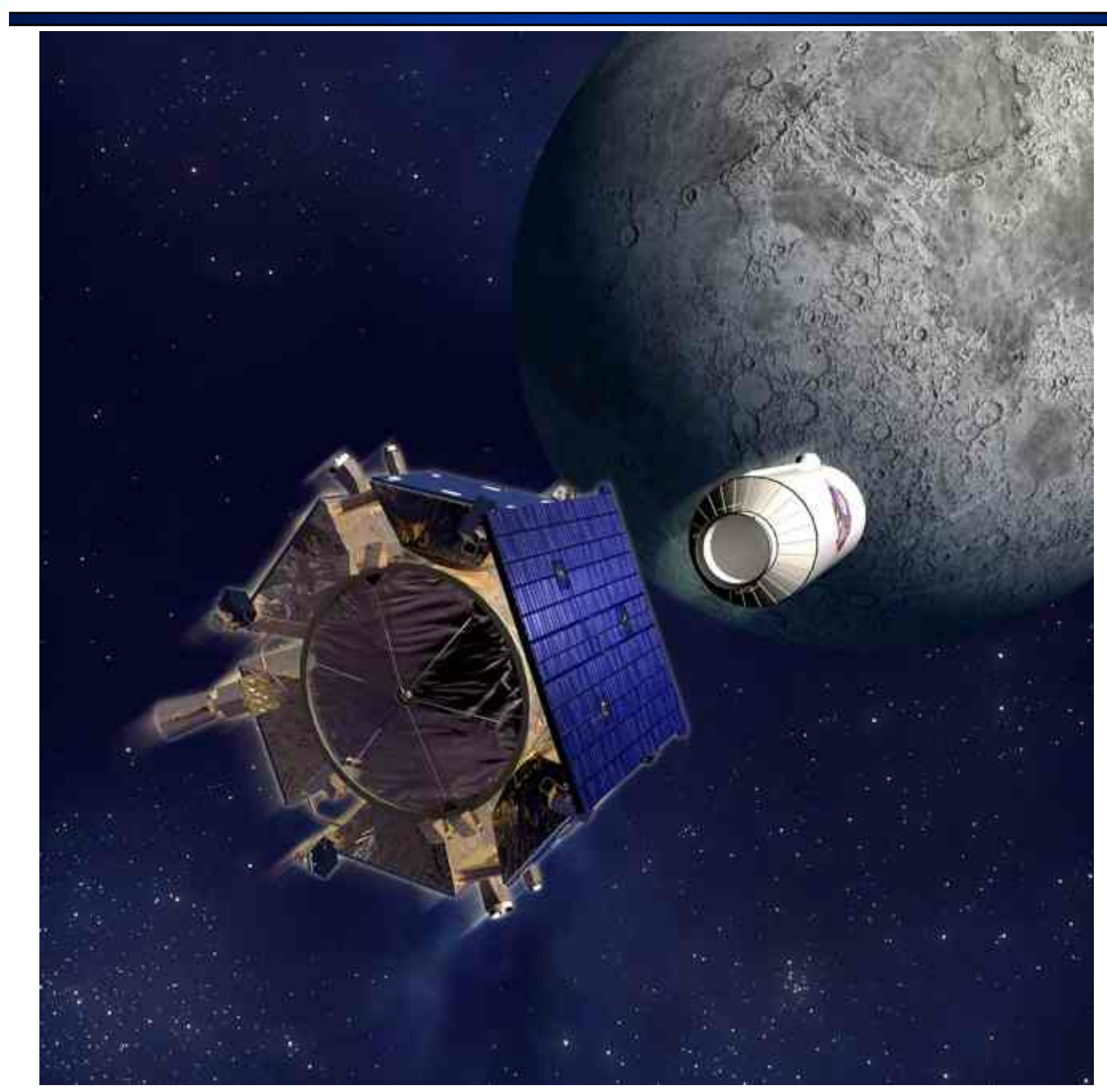




\section{Backup Slide - STA Noise}

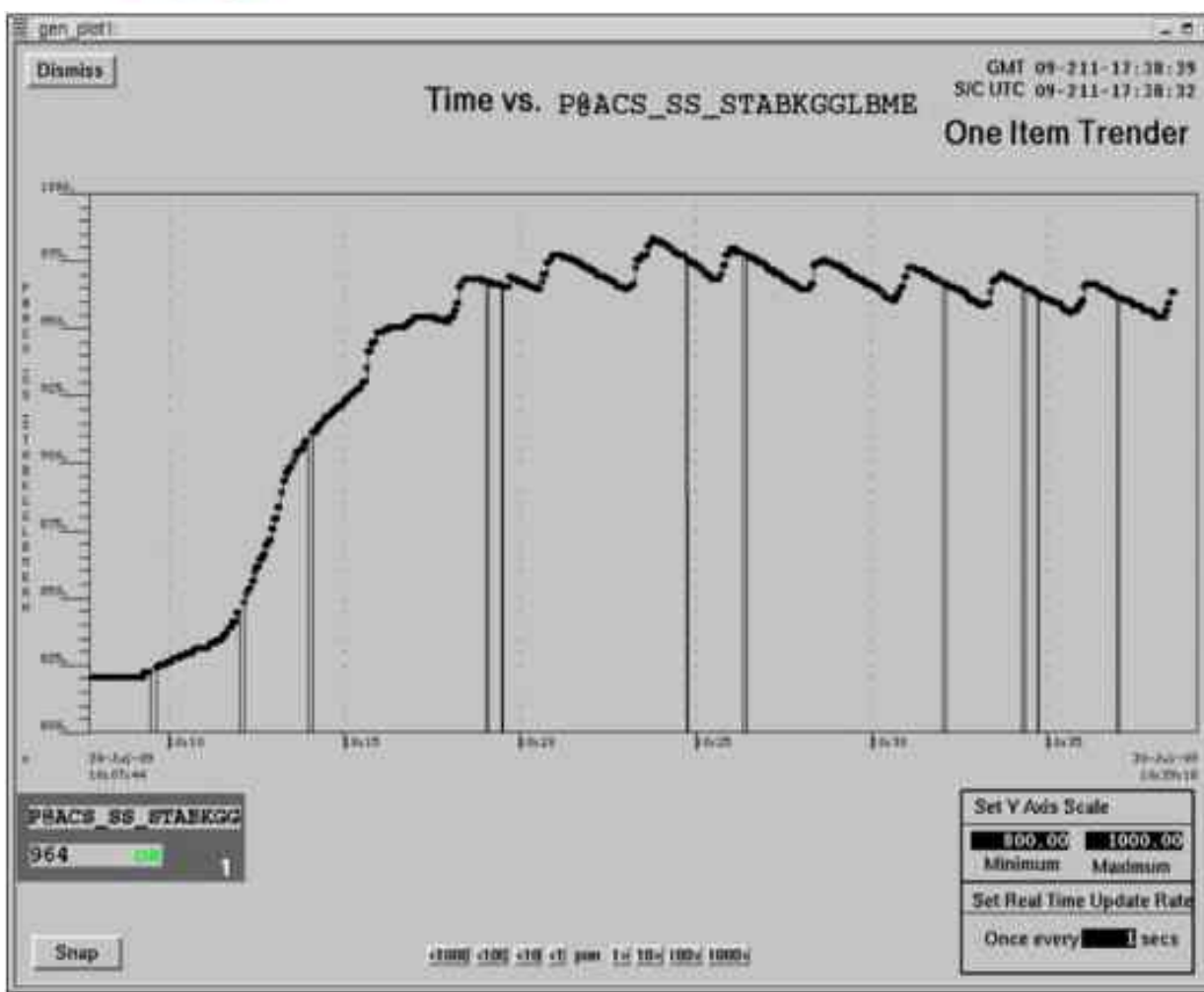

During Cold-Side Bakes, Star Tracker background noise levels corresponding to bake attitudes.

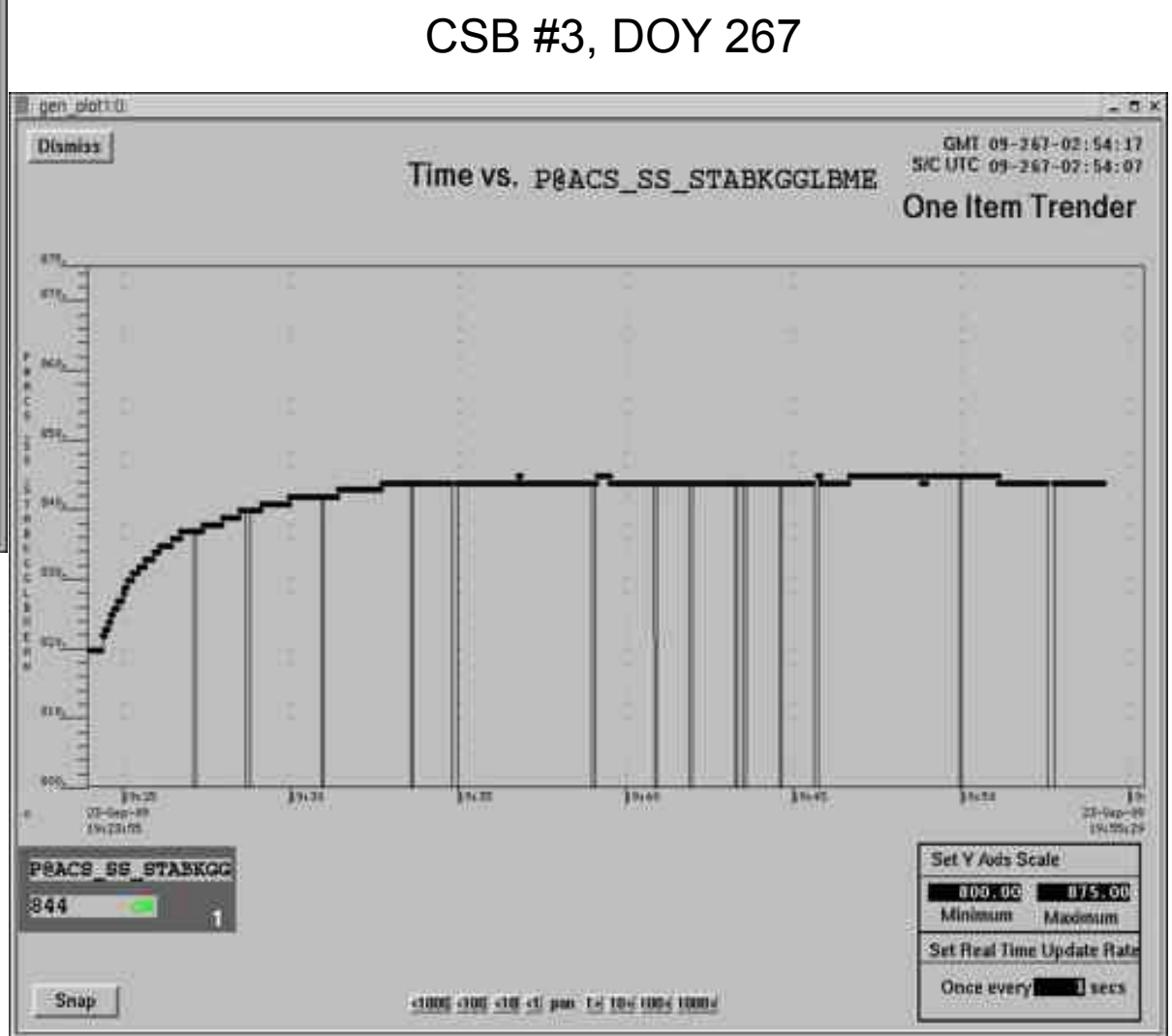

CSB \#2, DOY 211 


\section{Backup Slide - STA Spikes}

\section{During Cold-Side Bakes}
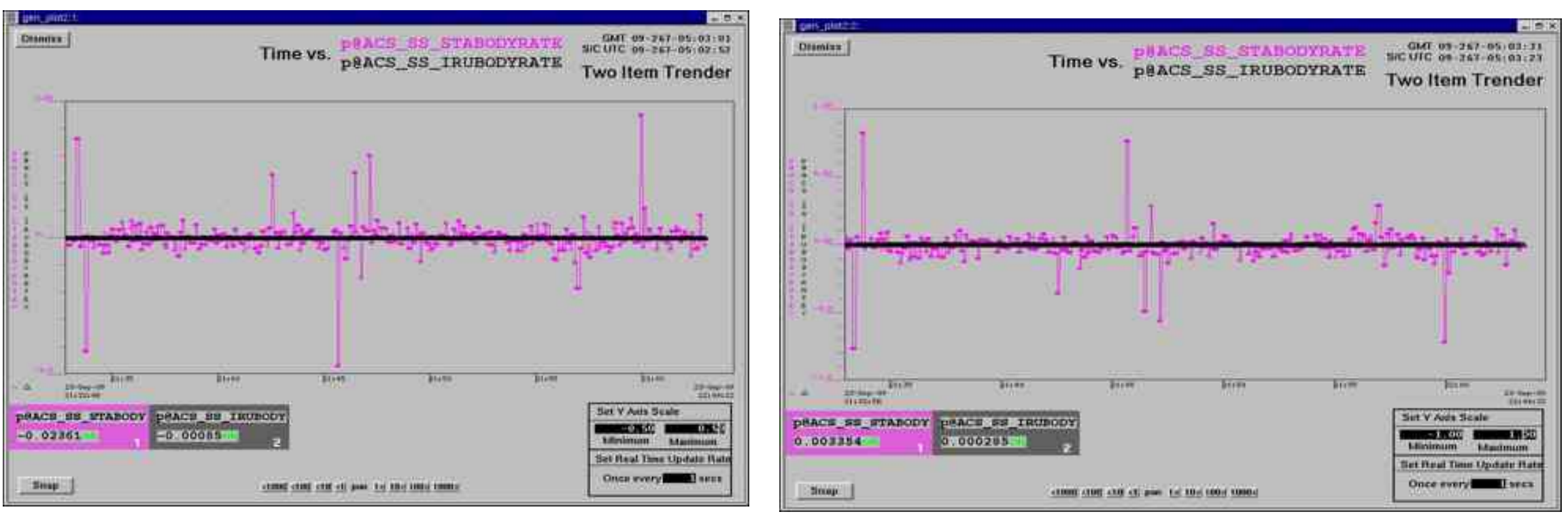

Also noted infrequent but large ( $1 \mathrm{deg} / \mathrm{sec})$ spikes in the STA rates which appear to be correlated with, but lag, attitude changes. Conjecture is that these may be attributable to debris in the STA FOV. 